\title{
Precision Sub-phenotyping of Histologically Stable Kidney Transplants by a Composite Molecular and Cellular Instability Score
}

\author{
D. Rychkov ${ }^{1,2}$, S. Sur ${ }^{1}$, M. Sirota ${ }^{*+2,3}$ and M. M. Sarwal ${ }^{*+1}$ \\ 1 Department of Surgery, Division of Multi-Organ Transplantation, UCSF, San Francisco, CA, USA \\ 2 Bakar Computational Health Sciences Institute, UCSF, San Francisco, CA, USA, \\ 3 Department of Pediatrics, UCSF, San Francisco, CA, USA \\ *marina.sirota@ucsf.edu and minnie.sarwal@ucsf.edu \\ + equal contribution
}

\begin{abstract}
Acute Rejection (AR) is the main cause of the graft dysfunction and premature graft loss, and diagnosis of rejection before advanced histological injury is crucial to salvage graft function. However, recent molecular studies have highlighted the unrecognized burden of sub-clinical graft rejection when graft function is preserved, and a dichotomy exists, of a histologically normal biopsy with molecular signatures of AR. Conversely, significant variation also exists in the definition of a stable allograft, defined as a transplant with absence of clinical AR, with absence of histological inflammation, though published studies have highlighted that some of these stable samples will not have stable immune quiescence, as they may be molecularly similar to AR. Thus, refining the definition of a stable allograft as one that is clinically, histologically and molecularly quiescent is critical, as the inclusion of stable allografts in mechanistic and clinical studies are vital to provide a normal, non-injured comparative group for all interrogative studies on understanding allograft injury.
\end{abstract}

With this goal in mind, we analyzed publicly available transcriptional data across 4,845 human kidney tissue samples from 38 Gene Expression Omnibus (GEO) datasets, inclusive of 510 allograft biopsy samples with AR, 1,154 renal allograft biopsies classified in each dataset as histological stable (hSTA), and 609 normal kidney (donor) samples. By applying a machine learning model, a substantive number of hSTA samples were found to be molecularly similar to $A R(m A R)$; these have been reclassified in this study as clinical and histological stable samples with transcriptional signatures overlapping with AR (hSTA/mAR), with the predominant expression of a subset of 6 genes (KLF4, CENPJ, KLF2, PPP1R15A, FOSB, TNFAIP3). To understand the cellular sources of these molecular signals, we utilized xCell, a cell type enrichment tool and interrogated 64 specific cell types to identify 5 (CD4+Tcm, CD4+ Tem, CD8+ Tem, NK cells, and Th1 cells) that were also highly predictive for classification of the AR phenotype in these studies. A combined gene and cell-type specific InstaScore (AUC 0.99) was developed using gene and cell subtype data to re-phenotype all hSTA allografts. This clearly defined two disparate hSTA biopsies: those that are both histologically and molecularly quiescent (hSTA/mSTA) or those that are histologically quiescent but molecularly similar to AR (hSTA/mAR). The clinical utility of the Instability Score was subsequently assessed by independent validation on a serial set of post-transplant hSTA biopsies, where strong significant correlation was observed between the score on 6 month post-transplant hSTA graft biopsies, where hSTA/mAR samples had a significant change in graft function and graft loss at 5 year follow-up. 
bioRxiv preprint doi: https://doi.org/10.1101/2019.12 18.881706; this version posted December 19,2019 . The copyright holder for this preprint (which was not certified by peer review) is the author/funder, who has granted bioRxiv a license to display the preprint in perpetuity. It is made available under aCC-BY-ND 4.0 International license.

In conclusion, our computational approach of precision sub-phenotyping of hSTA allografts by the InstaScore identifies discrepancies in the current recognition of a stable allograft by histology alone. Precision molecular sub-typing of the hSTA allograft into the hSTA/mSTA group is an important deliverable for selection of "true" STA samples for mechanistic studies, and into the hSTA/mAR group, for accurate prediction of subsequent patient clinical outcomes, and real time treatment stratification for hSTA/mAR allografts to positively impact long-term graft survival. 


\section{Introduction}

Breakthroughs in surgical approaches for organ transplantation and development of newer generations of immunosuppressive drugs to support engraftment across HLA barriers have resulted in a dramatic reduction in mortality among patients with an end stage organ disease, improvements in life quality and cognition, over the last decade[1]. However, overt and covert allograft acute rejection (AR) remains the most functionally relevant immune escape that drives adverse graft outcomes. Recent advances in omics technologies have uncovered the hidden burden of subclinical acute rejection (SAR), which can be observed even in the presence of an otherwise histologically pristine allograft [2,3]. Thus, precision molecular tissue phenotyping is needed to fully understand the granular phenotypes of clinical, histological and molecular injury from AR and to map clinical, histological and molecular quiescence for the true classification of a histologically stable (hSTA) allograft.

In this study, we propose that precision phenotyping of a kidney transplant biopsy by applying machine learning to gene expression measures which can enhance the accuracy of biopsy diagnosis [4]. The large amount of various molecular data in the public domain: genomic, transcriptomic, proteomic, and metabolomic, spurred by the expansion of next-generation sequencing technologies, coupled with advances in analytical and statistical methods, has already triggered the acceleration of the biological research in the field of personalized treatment and precision phenotyping in many other biomedical fields [5-7]. It now allows a unique opportunity to re-use aggregated human organ transplant related datasets in the Gene Expression Omnibus (GEO) [8], regardless of technology and platform era differences, utilizing novel approaches for data normalization and meta-analyses. Our group pioneered the metaanalysis of human biopsy transplant cohorts from four different solid organs - heart, kidney, liver, and lung - to identify and validate a common rejection module (CRM) of 11 genes (BASP1, CD6, CXCL10, CXCL9, INPP5D, ISG20, LCK, NKG7, PSMB9, RUNX3, and TAP1) that were significantly overexpressed in AR across all tissues, irrespective of organ source[9]. A similar approach utilized for 7 cross-organ tissue datasets confirmed a similar signature for AR (ISG20, CXCL9, CXCL10, CCL19, FCER1G, PMSE1, and UBD) [10], and 6 kidney transplant biopsy datasets were interrogated to identify an 85-gene signature associated with interstitial fibrosis and tubular atrophy [11]. Other meta-analysis studies on public data have been performed to study peripheral blood signatures of AR [12].

A significant obstacle in mining public transplant data is the difficulty in assigning what a truly stable transplant is, an issue of critical importance in this kidney of analysis, as this group of samples forms the control, "no-injury", immune-quiescent group for the analysis. Not only is there significant discrepancy (19-55\%) among pathologists for histological phenotyping [13,14], the histologic grading in renal transplant pathology is made within the Banff classification [15] which covers 10 different histologic lesions with an empirically derived, subjective scale of 0 to 3 , and alloantibody assessment and monitoring varies widely between institutions [16], resulting in considerable disagreements in the histological interpretation of what is a stable graft [17]. In a recent study [18], unsupervised archetypal analysis and reclassification of 1,208 biopsy samples resulted in $32 \%$ disagreement of transcriptional phenotypes with histological 
diagnosis [13,14]. In addition, work from our group and others [2,3,18-22] has confirmed that sub-clinical molecular inflammation and AR related transcriptional and protein signatures can exist in absence of obvious infiltrates by histology, and can predate full blown histological rejection and increase the risk of progressive chronic graft injury. Thus, the ability to truly define a "stable" allograft becomes a critical unmet need for clinical assessment of the allograft and for the conduct of interrogative mechanistic studies into allograft pathology and injury. Simply accepting all samples classified as "stable" from different investigators and datasets will neglect the important biological diversity that naturally exists in the histological definition of a "stable" sample and will necessarily lead to false positive and false negative discoveries when attempting to study graft rejection.

We hypothesize that accurate classification of a stable allograft is not only a critical requirement for the conduct of successful meta-analyses of transplant injury, but also, more precise (sub-)phenotyping and early recognition of AR of otherwise functionally and histologically stable allografts on protocol biopsies, will allow timely and proactive adjustment of immunosuppression to immunologic risk, with a positive impact on graft survival and the patient's quality of life $[5,17]$. To enable this, we have leveraged the largest public dataset analyzed in human renal transplantation: 4,845 kidney tissue microarray samples from 38 publicly available human normal kidney and transplant kidney tissue datasets, run on 18 different microarray platforms, to investigate the molecular diversity of histologically stable allografts. This study provides a new composite gene and cell specific analysis tool, the Instability Score (or InstaScore), that could provide an important adjunct to a pathologist for comprehensive and highly quantitative phenotyping of a protocol kidney transplant biopsy, in addition to the standard histological assessment. 


\section{Methods and Materials}

Data collection. We carried out a comprehensive search for publicly available microarray data at NCBI GEO (Gene Expression Omnibus) database [8] (http://www.ncbi.nlm.nih.gov/geo/). We used the keywords "kidney", "renal", "transplant", and "biopsy", among organisms "Homo Sapiens" and study type "Expression profiling by array". We identified and collected publicly available microarray data from 38 datasets with a total of 4,845 human kidney biopsy samples (Table 1). For each dataset, we carefully collected the platform information, annotations, and expression data. The gene expression measurements were made on 18 different platforms (Table 1). The commercial chips were designed by the manufacturers Affymetrix, Agilent and Illumina, and the custom chips were by Functional Genomics Facility at Stanford University and Bioinformatics and Gene Network Research Group at Zhejiang University. The number of probes varied widely from 1,347 to 54,675 . In order to preserve as many genes as possible for further analysis we filtered out two datasets GSE26578 and GSE1563 with the lowest number of probes. We also filtered out 5 more datasets: GSE1743, GSE21785, GSE98320, GSE83486, and GSE109346, due to multiple absent or poor annotations such that it was impossible to confidently identify phenotypes associated with the individual samples. The dataset GSE343 was removed since the data of only log 2 ratios of intensities in Cy5 and Cy 3 channels were available. We found two datasets, GSE14700 and GSE82337, and one sample from GSE36059 with very sparse expression data and significant percentage of missing values, hence we filtered out these datasets as well. In the last step, we examined all datasets for any duplicate samples since many studies recycle previously published data. We were able to identify and remove 179 duplicate samples from GSE11166, GSE14328, GSE34437, GSE50058, and GSE72925 based on available metadata. We then used an R package DoppelgangR [23] that is based on calculations of correlations between samples and manually curated its results for any false positives and identified 257 samples as highly possible duplicates that were then also removed. Using the PCA plots, boxplots, and density plots, we analyzed the datasets for any presence of outliers. After stringent data quality control procedures, we composed the final dataset consisted of 28 studies with 2,273 samples. Their diagnostic annotations included 510 Acute rejection (AR) (including Antibody mediated rejection (ABMR), T cell mediated rejection (TCMR), AR, AR with Chronic allograft nephropathy ( $A R+C A N)$, Borderline rejection (BL), BL+CAN, Mixed rejection), 1,154 Stable (STA), and 609 Normal (i.e. biopsy taken prior to transplantation). The summary for the collected studies is represented in Table 1.

\begin{tabular}{|c|c|c|c|c|c|c|c|c|c|}
\hline Study & PMID & Platform & Probes & Total & AR & STA & Normal & Year & Country \\
\hline GSE343* & 12853585 & GPL271 LC-17 \& GPL272 LC-20 & $\begin{array}{c}28032 \& \\
37632\end{array}$ & 41 & 26 & 0 & 15 & 2003 & USA \\
\hline GSE1563* & 15307835 & GPL8300 [HG_U95Av2] Affymetrix Human Genome U95 Version 2 & 12625 & 31 & 7 & 10 & 9 & 2004 & USA \\
\hline GSE1743* & 15476476 & GPL96 [HG-U133A] Affymetrix Human Genome U133A & 22283 & 41 & 0 & 41 & 0 & 2004 & USA \\
\hline GSE7392 & 17397532 & GPL570 [HG-U133_Plus_2] Affymetrix Human Genome U133 Plus 2.0 & 54675 & 22 & 0 & 7 & 15 & 2007 & USA \\
\hline GSE9493 & 19017305 & GPL570 [HG-U133_Plus_2] Affymetrix Human Genome U133 Plus 2.0 & 54675 & 56 & 21 & 21 & 14 & 2007 & Switzerland \\
\hline GSE10419 & 20525436 & GPL887 Agilent-012097 Human 1A Microarray (V2) G4110B & 22153 & 29 & 0 & 29 & 0 & 2008 & Japan \\
\hline GSE11166 & 19443638 & GPL570 [HG-U133_Plus_2] Affymetrix Human Genome U133 Plus 2.0 & 54675 & 49 & 1 & 27 & 21 & 2008 & USA \\
\hline GSE14328 & 20150539 & GPL570 [HG-U133 Plus_2] Affymetrix Human Genome U133 Plus 2.0 & 54675 & 36 & 18 & 18 & 0 & 2009 & USA \\
\hline
\end{tabular}


bioRxiv preprint doi: https://doi.org/10.1101/2019.12.18.881706; this version posted December 19, 2019. The copyright holder for this preprint (which was not certified by peer review) is the author/funder, who has granted bioRxiv a license to display the preprint in perpetuity. It is made available under aCC-BY-ND 4.0 International license.

\begin{tabular}{|c|c|c|c|c|c|c|c|c|c|}
\hline GSE14700* & 20713790 & $\begin{array}{l}\text { GPL8150 Stanford Microarray Functional Genomics Homo sapiens } \\
\qquad 34.6 \mathrm{~K}\end{array}$ & 34599 & 40 & 0 & 0 & 40 & 2009 & Austria \\
\hline GSE21374 & 20501945 & GPL570 [HG-U133_Plus_2] Affymetrix Human Genome U133 Plus 2.0 & 54675 & 282 & 76 & 206 & 0 & 2010 & Canada \\
\hline GSE22459 & 20813870 & GPL570 [HG-U133_Plus_2] Affymetrix Human Genome U133 Plus 2.0 & 54675 & 25 & 0 & 25 & 0 & 2010 & USA \\
\hline GSE25902 & 21881554 & GPL570 [HG-U133_Plus_2] Affymetrix Human Genome U133 Plus 2.0 & 54675 & 88 & 27 & 37 & 24 & 2010 & USA \\
\hline GSE26578* & - & GPL9301 Zhejiang university human 449 oligonucleotide array & 1347 & 85 & 34 & 51 & 0 & 2011 & China \\
\hline GSE30718 & 22343120 & GPL570 [HG-U133_Plus_2] Affymetrix Human Genome U133 Plus 2.0 & 54675 & 19 & 0 & 11 & 8 & 2011 & Canada \\
\hline GSE34437 & 23437201 & GPL570 [HG-U133_Plus_2] Affymetrix Human Genome U133 Plus 2.0 & 54675 & 36 & 17 & 7 & 12 & 2011 & USA \\
\hline GSE34748 & 22335458 & GPL570 [HG-U133_Plus_2] Affymetrix Human Genome U133 Plus 2.0 & 54675 & 36 & 0 & 36 & 0 & 2011 & USA \\
\hline GSE36059 & 23356949 & GPL570 [HG-U133_Plus_2] Affymetrix Human Genome U133 Plus 2.0 & 54675 & 410 & 122 & 280 & 8 & 2012 & Canada \\
\hline GSE43974 & 25427168 & GPL10558 Illumina HumanHT-12 V4.0 expression beadchip & 47323 & 463 & 0 & 112 & 351 & 2013 & Netherlands \\
\hline GSE44131 & 24030736 & GPL6244 [HuGene-1_0-st] Affymetrix Human Gene 1.0 ST Array & 29096 & 12 & 0 & 12 & 0 & 2013 & USA \\
\hline GSE47097 & 23763497 & GPL6883 Illumina HumanRef-8 v3.0 expression beadchip & 47323 & 40 & 36 & 4 & 0 & 2013 & Netherlands \\
\hline GSE48581 & 23915426 & GPL570 [HG-U133_Plus_2] Affymetrix Human Genome U133 Plus 2.0 & 54675 & 306 & 78 & 222 & 6 & 2013 & Canada \\
\hline GSE50058 & 24127489 & GPL570 [HG-U133_Plus_2] Affymetrix Human Genome U133 Plus 2.0 & 54675 & 56 & 31 & 25 & 0 & 2013 & USA \\
\hline GSE50084 & 26484130 & GPL6244 [HuGene-1_0-st] Affymetrix Human Gene 1.0 ST Array & 29096 & 48 & 28 & 20 & 0 & 2013 & USA \\
\hline GSE52694 & 26176825 & GPL10558 Illumina HumanHT-12 V4.0 expression beadchip & 23719 & 14 & 0 & 14 & 0 & 2013 & $\begin{array}{l}\text { Czech } \\
\text { Republic }\end{array}$ \\
\hline GSE53605 & 24698514 & GPL571 [HG-U133A_2] Affymetrix Human Genome U133A 2.0 & 22277 & 31 & 13 & 18 & 0 & 2013 & USA \\
\hline GSE53769 & 20819195 & GPL16686 [HuGene-2_0-st] Affymetrix Human Gene 2.0 ST Array & 53617 & 28 & 0 & 10 & 18 & 2013 & Austria \\
\hline GSE54888 & - & GPL6244 [HuGene-1_0-st] Affymetrix Human Gene 1.0 ST & 33252 & 54 & 0 & 0 & 54 & 2014 & Brazil \\
\hline GSE57387 & 27452608 & GPL5175 [HuEx-1_0-st] Affymetrix Human Exon 1.0 ST & 17881 & 104 & 0 & 104 & 0 & 2014 & USA \\
\hline GSE60807 & 26908771 & $\begin{array}{l}\text { GPL6480 Agilent-014850 Whole Human Genome Microarray 4x44K } \\
\text { G4112F }\end{array}$ & 41093 & 37 & 0 & 0 & 37 & 2014 & Austria \\
\hline GSE65326 & 25307039 & GPL10558 Illumina HumanHT-12 V4.0 expression beadchip & 47323 & 6 & 0 & 6 & 0 & 2015 & Australia \\
\hline GSE69677 & 27369853 & $\begin{array}{l}\text { GPL14951 Illumina HumanHT-12 WG-DASL V4.0 R2 expression } \\
\text { beadchip }\end{array}$ & 29360 & 76 & 24 & 0 & 52 & 2015 & Italy \\
\hline GSE72925 & 27140517 & GPL570 [HG-U133_Plus_2] Affymetrix Human Genome U133 Plus 2.0 & 54675 & 46 & 10 & 36 & 0 & 2015 & USA \\
\hline GSE76882 & 26990570 & GPL13158 [HT_HG-U133_Plus_PM] Affymetrix HT HG-U133+ PM & 17564 & 182 & 83 & 99 & 0 & 2016 & USA \\
\hline GSE82337* & 27225518 & GPL10558 Illumina HumanHT-12 V4.0 expression beadchip & 47323 & 78 & 0 & 78 & 0 & 2016 & USA \\
\hline GSE83486* & 28436117 & GPL10558 Illumina HumanHT-12 V4.0 expression beadchip & 47323 & 60 & $?$ & $?$ & 0 & 2016 & Norway \\
\hline GSE98320* & 28614805 & GPL15207 [PrimeView] Affymetrix Human Gene Expression Array & 49395 & 1208 & $?$ & $?$ & 0 & 2017 & Canada \\
\hline GSE109346* & - & GPL10558 Illumina HumanHT-12 V4.0 expression beadchip & 47323 & 26 & 0 & 26 & 0 & 2018 & $\begin{array}{c}\text { Czech } \\
\text { Republic }\end{array}$ \\
\hline
\end{tabular}

Table 1. Datasets collected from Gene Expression Omnibus (GEO).

* Datasets not included into this study

Data processing and normalization. Several pre-processing steps were applied prior to the main analysis. Raw fluorescence intensity data stored in .CEL or .txt files were downloaded and preprocessed depending on the platform. The data processing included background correction, log2 transformation, quantile normalization and probe to gene mapping using $\mathrm{R}$ language version 3.5.1 [24]. For the Affymetrix platform, we used the R package SCAN.UPC [25] available at Bioconductor [26] (http://ww.bioconductor.org). In contrast to some other popular multiarray normalization algorithms like RMA [27] that estimate probe-level effects and standardize variances across arrays based on the information from a whole dataset, SCAN.UPC is a singlearray method that normalizes every sample independently from other samples. This is considered as an advantage [25] since this approach is robust to any influence from possible 
outliers in the data. The database for the mapping between probes and Entrez gene IDs were taken from the BrainArray resource [28] version 22

(http://brainarray.mbni.med.umich.edu/Brainarray/Database/CustomCDF/22.0.0/entrezg.asp).

For the Agilent and Illumina platforms, we downloaded non-normalized raw data and performed data processing using neqc() function within limma package [29] from Bioconductor. This algorithm [30] estimates parameters based on normal-exponential (normexp) convolutional model with joint likelihood estimation and with the help of negative control probes. The offset 16 was added to the intensities after the background adjustment by default, as it was shown as the most optimal value to improve FDR of the normexp algorithm [29]. However, some data from the Agilent and Stanford platforms did not contain any negative control probes. Therefore, we used similar processing steps manually to reproduce the methodology by applying backgroundCorrect() function from limma package by the mle normexp method with offset 16. Log2 transformation and quantile normalization was performed after this. The probe-gene mapping was implemented using the information from biomaRt database [31] or GPL files.

To gain additional statistical power from the large dataset we were able to collect, we chose to merge all the studies to perform a meta-analysis. In order to merge data from different studies and different platforms we had to first correct for potential batch effects. There are a number of papers that address this issue [32-36], but unfortunately, a one-fits-all solution doesn't exist. Different normalization methods have their advantages and disadvantages [37-39] in removing batch effects, however, they can also become a critical problem in correcting the imbalanced data [32,36]. We examined the performance of several approaches that included ComBat [40] as a part of sva package [41], Quantile Normalization (QN) [42], Remove Unwanted Variation (RUV) [43] and Harman [44]. We identified superior batch effect minimization with ComBat in comparison to other methods and used it to normalize the data. A short description of our examinations can be found in Supplemental materials. The process of data aggregation, normalization and merging is schematically described in Fig 1a. 


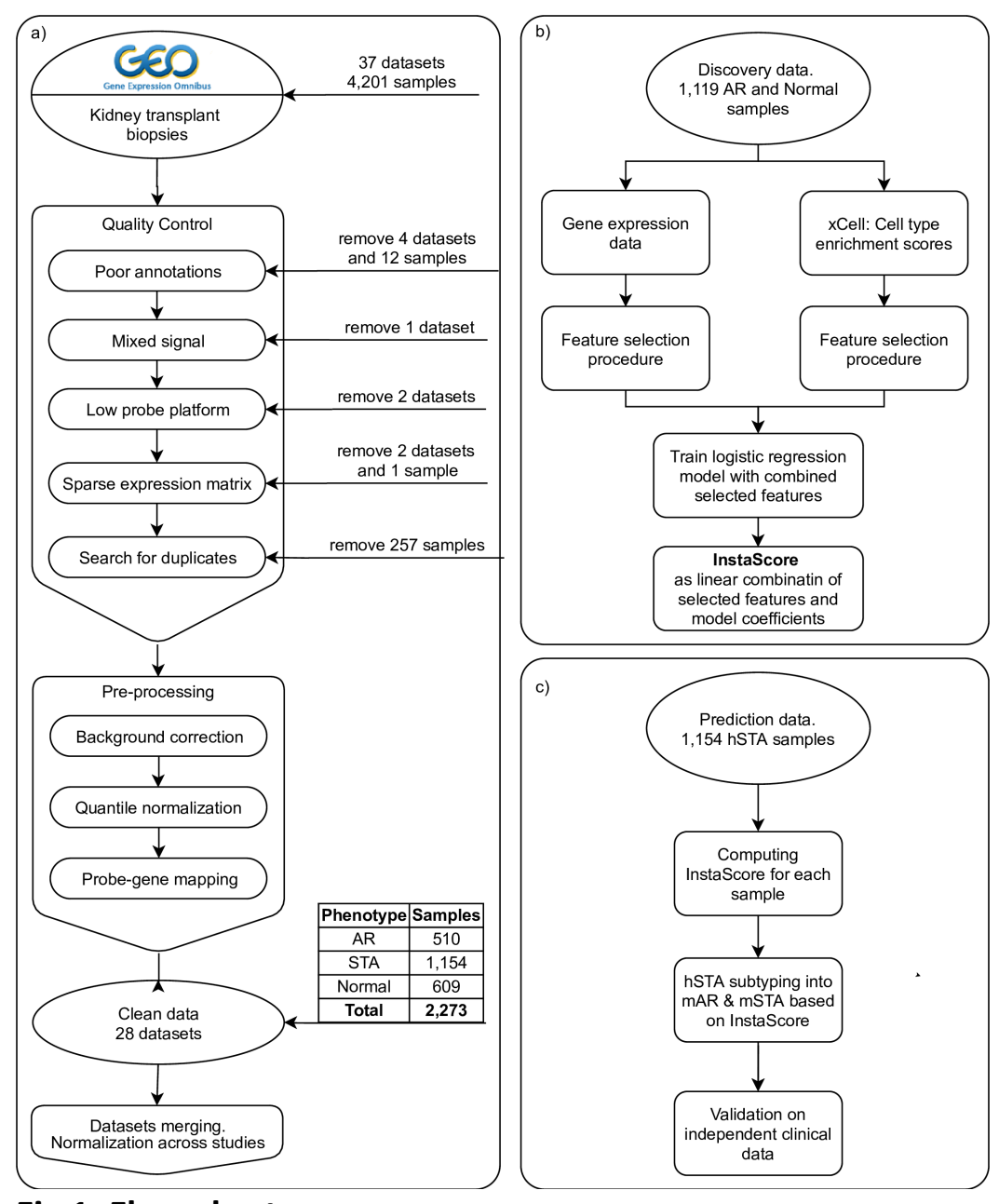

Fig 1. Flow chart

(a) Data pre-processing and Normalization. (b) Feature selection and creating the Instability Score (InstaScore). (c) Applying InstaScore to hSTA for sub-phenotyping and validating results.

Differentially Expressed Gene (DEG) analysis. To identify differentially expressed genes in the first analysis AR vs Normal we used the Significance Analysis of Microarrays (SAM) [45] method that was implemented in the $R$ package siggenes [46]. We utilized the false discovery rate (FDR) [47] with Benjamini-Hochberg procedure [48] for multiple testing correction and use the adjusted cutoff of 0.05 . For the second level of significance, we selected only those genes that have the fold change greater than 1.5.

Pathway Analysis. We leveraged the Gene Ontology database using the gene set enrichment analysis implemented in the R package clusterProfiler [49] to perform functional annotations for the significantly up- and down-regulated genes. We used FDR multiple correction method with the enrichment significance cut-off at level 0.05. For the gene network analysis, we utilized the STRING protein-protein association networks database [50] (https://string-db.org).

Cell type enrichment analysis. In order to estimate the presence of certain cell types in biopsy samples, we leveraged a recently published cell type enrichment tool xCell [51]. xCell leverages gene expression data from microarray and RNA-seq experiments and is used to perform 
enrichment analysis for up to 64 immune and stromal cell types. We focused on 34 immune related and 11 non-immune cell types (Table S1) that we selected manually as relevant to the transplant injury process. In our analysis, we used a dedicated R package that is available on the author's GitHub account for this purpose. This analytical method is a gene signatures-based method and converts the gene expression into cell type enrichment scores. The authors especially emphasize that this is not a deconvolution method that provides percentage of cell types containing in a tissue but rather the enrichment tool allowing to compare samples for each cell type but not otherwise. The enrichment scores for each cell type were used to compare AR and Normal samples and to identify cell types that are significantly different in individuals with AR as opposed to normal controls by performing the non-parametric twosample Mann-Whitney-Wilcoxon statistical test. We utilized the multiple testing correction by using the Benjamini-Hochberg method. Adjusted $p$-value $<0.05$ was used as the threshold.

Feature selection procedure. In our efforts to select the most important features in distinguishing AR vs Normal samples, we performed the following steps. First, we split the whole data into training and testing sets in the ratio 80:20 and performed all feature selection procedures on the training set with benchmarking on the testing set. After identifying the significant features from a statistical test described above on the training set, we searched for features that correlate with the outcome no less than $2 / 3$ of a maximum correlation value. In the final step, we applied Recursive Feature Elimination (RFE) technique with Random Forest (RF) model from the R package caret [52]. We used 5-fold cross validation (CV) technique with 100 repeats. To benchmark the model, we used the area under the ROC curve that is more suitable for data with some disbalance in outcome - in our case the ratio AR:Normal is 0.84 . To decrease the bias of random split as well as to avoid the model overfitting, we also introduced the tolerance of $1 \%$ to the feature selection mechanism, i.e. the algorithm was choosing the simplest model with the smallest number of features that performs within range $99-100 \%$ of the best model.

To perform the steps described above, we adopted the R package fese $R$ [53] and modified it to implement the parallel computations, the AUROC metric for model benchmarking, and the tolerance parameter of model performance. After the feature selection steps, we benchmarked the features with RF model on the testing set.

Instability Score and hSTA sub-phenotyping. The method of sub-phenotyping hSTA samples is formed on creating a scoring system based on selected features and scoring the hSTA samples. Based on the score values, histologically STA samples are identified as molecularly AR or molecularly STA. We denoted this split as hSTA/mAR and hSTA/mSTA, respectively.

For our current analysis, two types of data were available: gene expression data and cell type enrichment scores (obtained computationally using xCell). Based on these two data types, we performed the feature selection procedure described above to find sets of genes and cell types that highly associated with AR. Next, we z-scaled each feature, a gene or a cell type, and built a logistic regression model with all features to identify feature importance as model coefficients. Using these coefficients, we created a linear formula to compute a score, that we called the Instability Score (or InstaScore): 


$$
\begin{aligned}
\text { InstaScore }= & 0.596+2.096 \times \mathrm{KLF} 4+2.534 \times \mathrm{CENPJ}+0.311 \times \mathrm{KLF} 2 \\
& +1.447 \times \mathrm{PPP} 1 \mathrm{R} 15 \mathrm{~A}-1.633 \times \mathrm{FOSB}+0.268 \times \mathrm{TNFAIP} 3 \\
& +2.249 \times \mathrm{NK} \text { cells }+0.542 \times \mathrm{CD} 4+\mathrm{T}_{\mathrm{cm}} \text { cells }+0.833 \times \mathrm{CD} 4+\mathrm{T}_{\mathrm{em}} \text { cells } \\
& +0.709 \times \mathrm{CD} 8+\mathrm{T}_{\mathrm{em}} \text { cells }+0.146 \times \mathrm{Th}_{1} \text { cells. }
\end{aligned}
$$

The positive InstaScore values separate AR from Normal samples which obtain negative values. By following the same steps, we then computed the InstaScore for the histologically STA samples and applied the zeroth threshold to obtain the split into mAR and mSTA subtypes. This whole approach is schematically represented in the form of the flow chart in the Figs $1 \mathrm{~b}$ and $\underline{1 \mathrm{c}}$.

\section{Results}

In the current study, we aimed to identify and characterize a phenotypic split of histologically STA (hSTA) kidney allografts that redefine inherent biological and functionally relevant differences that underlie the simple classification of a stable allograft by clinical function or histology alone, recognizing the redundancy of kidney reserve and the inherent discrepancies between different pathologists to interpret histology. In addition to better phenotyping hSTA allograft variabilities we also wanted to develop a robust, unbiased, quantitative classification method that could be integrated into clinical care, to better define immunologically quiescent renal allograft health. We leveraged all up-to date publicly available kidney biopsy microarray data and performed a feature selection procedure based on the Random Forest algorithm to identify a subset of genes and cell types that better distinguish AR and Normal samples. We then combined the feature selected genes and cell types into one score value, called InstaScore, applied it to hSTA samples, and identified the molecular subtype separation onto hSTA/mAR and hSTA/mSTA (Fig 1C). The prediction performance of the InstaScore was developed on public data and independently validated transcriptional data with linked clinical measurements.

Datasets and Study Cohort. As a result of the data search in NCBI GEO and filtering procedures (see Methods and Materials) we were able to identify a robust dataset consisting of 2,273 samples including 510 AR, 1,154 STA, and 609 Normal samples from a total of 28 independent studies (Table 1$)$. In the collection of the data on the Acute Rejection samples we included original annotations with investigator-based diagnoses of Antibody Mediated Rejection (ABMR), T-cell Mediated Rejection (TCMR), Acute Rejection with Chronic Allograft Nephropathy $(A R+C A N)$, Borderline rejection (BL), BL+CAN, and Mixed rejection (TCMR and ABMR). Stable (STA) samples were histologically defined by each dataset as allografts not having rejection or any abnormalities including inflammation and fibrotic formations at the time of biopsy according to Banff's classification criteria [15]. Normal samples were defined as donor engraftment biopsies at the time of transplantation.

\section{Differential Gene Expression Analysis Identifies Up-regulation of Immune Related Pathways in Rejection. Though prior gene expression studies have been done on understanding transcriptional perturbations in AR [54-57], leveraging thousands of kidney biopsy samples from public data gives us the statistical power to discover small biological effects during transplant rejection and injury and utilize this signature to unravel biological and molecular differences within hSTA transplants. In order to prepare all collected data in the form of separate studies, several preprocessing steps were carried out (see Methods and Materials).}


The scatter plots of the first two principal components of the gene expression data before and after the batch correction shows successful removal of batch effects (Fig S1). Next, we performed differential gene expression analysis AR vs Normal on the data (Benjamini-Hochberg adjusted p-value 0.05 and fold change 1.3) and identified 1,509 significantly differentially expressed genes including 848 up- and 661 down-regulated genes (Table S2). Further hierarchical clustering analysis on the significant genes based on Ward's clustering technique was performed and showed the significant separation ( $p=1.5 \mathrm{E}-14)$ between classes (Fig 2a). In addition, principal component analysis (PCA) confirmed the class separation (Fig S2). By leveraging the Gene Ontology database with the gene set enrichment analysis implemented in the $\mathrm{R}$ package clusterProfiler [49], we performed functional annotation for the significant genes and found, as expected, up-regulated genes were enriched in the regulation of the immune response, cell aggregation and activation, and innate immunity (Fig S3a). The down regulated genes were enriched in metabolic processes (Fig S3c). We also leveraged STRING, a tool for the functional protein association network analysis, [58] (https://string-db.org) for both up- and down-regulated genes showing significant connectivity between the sets of genes (Figs S3b, $\underline{\mathrm{S} 3 \mathrm{~d})}$.
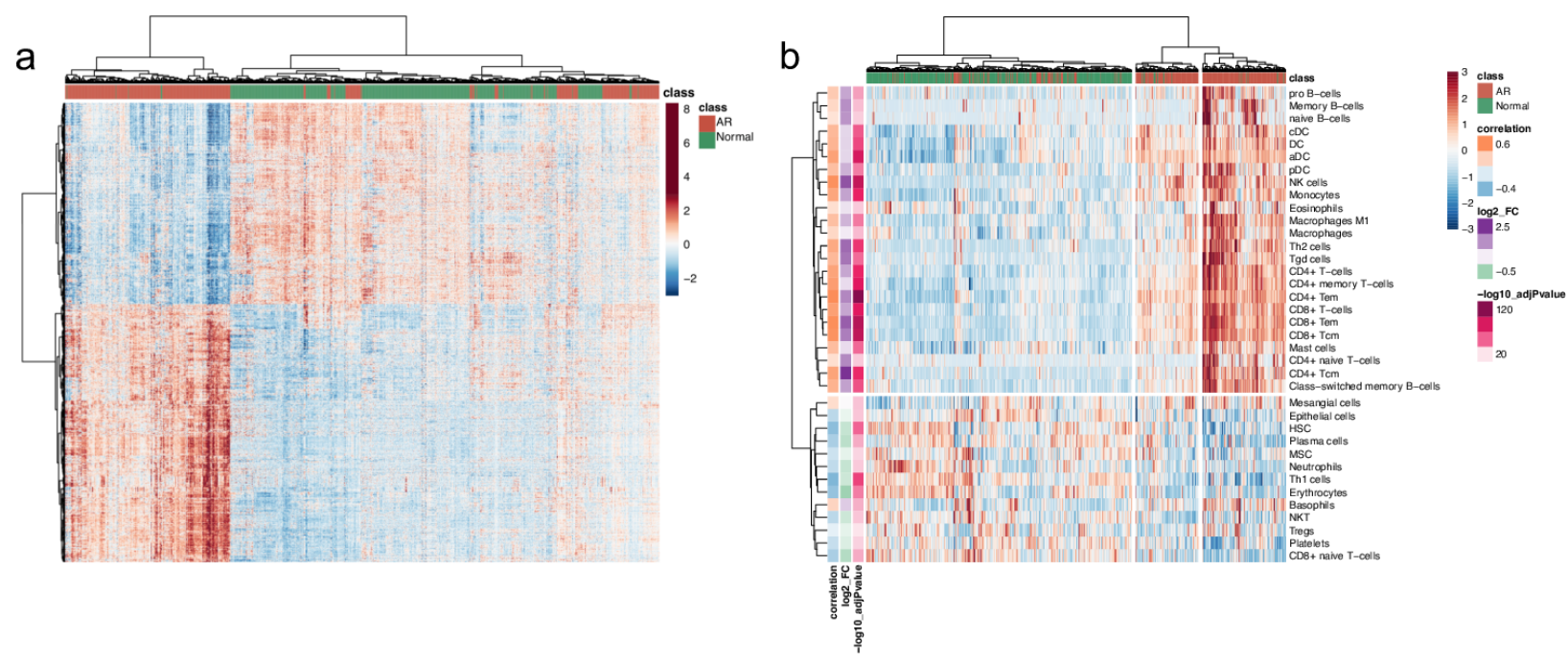

Fig 2. The heatmap plots for differentially expressed genes and significantly enriched cell types.

(a) Heatmap clustering plot for significant genes from SAM analysis of AR vs Normals. (b) Heatmap clustering plots for significant cell types from the non-parametric Wilcoxon statistical test $(\mathrm{BH} \mathrm{p}$-adj $<0.05)$ in the analysis of AR vs Normals.

\section{Cell Type Enrichment Analysis Identifies Immune Cell Types Associated with Acute Rejection.}

To highlight the biological heterogeneity and to capture signals from infiltrating cell type specific effects in injured and stable kidney transplants, we performed cell type enrichment analysis that leverages cell type frequencies from the gene expression data. Currently, though single cell sequencing approaches are being developed, they are expensive, especially for large cohorts of this size, and often left over, or adequately preserved tissue is not available. Cell type deconvolution and enrichment methods provide an opportunity to look computationally at the cellular infiltration and activation and provide a unique assessment for STA allografts that may 
have subtle and sub-clinical rejection, not detectable by standard pathology. The xCell tool [51] computes enrichment scores for 64 immune and stroma cell types by leveraging tissue gene expression data. In our analysis, we focused on 45 cell types (Table S1) that would be more relevant for organ transplant. We performed non-parametric Wilcoxon tests to identify cell types that are significantly differentially enriched between AR and Normal samples. The pvalues were adjusted to multiple testing correction with Benjamini-Hochberg method and with significant threshold of 0.05 . We found 25 cell types (the majority of them are Lymphoid and Myeloid cells) that are significantly enriched in Acute Rejection, and 12 cell types (Immune, Stromal cells, and Hematopoietic stem cells) that are enriched in Normal kidneys. We show these results along with computed effect sizes and correlations with the case-control status in the form of the heatmap plot in the Fig $2 \mathrm{~b}$. On the heatmap, it is also interesting to see that AR samples fall into two main sub-clusters $(p=1.2 \mathrm{e}-10)$ : one was mostly enriched in Lymphocytes, NK cells and Macrophages in contrast to another which had minimal Lymphocytes activation, and may represent temporal differences in rejection evolution or recovery. We observe that $B$ cells, Dendritic cells, Macrophages, and T-cells form cell type specific sub-clusters that show the coordinated activation of the immune cells in kidney tissues. These results are in agreement with previous observations [54] that have shown AR sub-phenotypical splits by genes expression and cell types. Unsupervised clustering of hSTA along with AR and Normal samples revealed their heterogeneity hinting that some histologically stable samples have molecular signal closer to AR samples (Fig S4).

Leveraging Feature Selection Procedure to Optimize AR classification. Following the feature selection procedure (Methods), we dramatically decrease the number of model features from all 1,509 differentially expressed genes only 6 pivotal upregulated genes: KLF4, CENPJ, KLF2, PPP1R15A, FOSB, TNFAIP3 (AUC 0.98; Fig S5a), genes enriched as zinc finger proteins and expressed mostly in CD33+ Myeloid cells, and 5 cell types from the original set of 37 differentially enriched cell types: $C D 4+T c m, C D 4+T e m, C D 8+T e m, N K$ cells, and Th1 cells, with CD4+ Tcm having the largest effect size in this model (AUC 0.92; Fig S5b).

The feature selected cell types show a predominant role for infiltration and activation of effector T cells and NK cells in AR, and the feature selected genes appear to have broad cellular functions in AR, triggered by mononuclear activation and infiltration and collectively driving a variety of functions such as DNA recognition, RNA packaging, transcriptional activation and regulation of apoptosis. Interestingly, though previously the set of 11 genes in common rejection module (CRM) [9] identified from our cross organ (kidney, heart, liver, lung) metaanalysis study of transplant rejection is enriched in this current analysis, none of them make it to this final minimal feature selection set, suggesting this current 6 gene-set maybe more specific for absence of AR in the renal allograft, as the precise definition of a hSTA/mSTA allograft was not available in the earlier analysis.

A generated Random Forest classification model for these 6 genes and 5 cell types, internally validated using 5 -fold cross-validation with 100 repeats, obtained an AUC of 0.98 (sensitivity 0.94, specificity 0.94) for the genes alone and an AUC of 0.92 (sensitivity 0.85 , specificity 0.88 ) for the cell types for identification of a tissue sample with histologically confirmed AR (Fig 3a). We furthermore combined the feature selected genes and cell types into one score value, 
called InstaScore (Methods). By utilizing the InstaScore, we were able to perform the split into AR and Normal samples with slightly improved AUC 0.99 with sensitivity 0.95 and specificity 0.94 (Figs 3b, 3c).
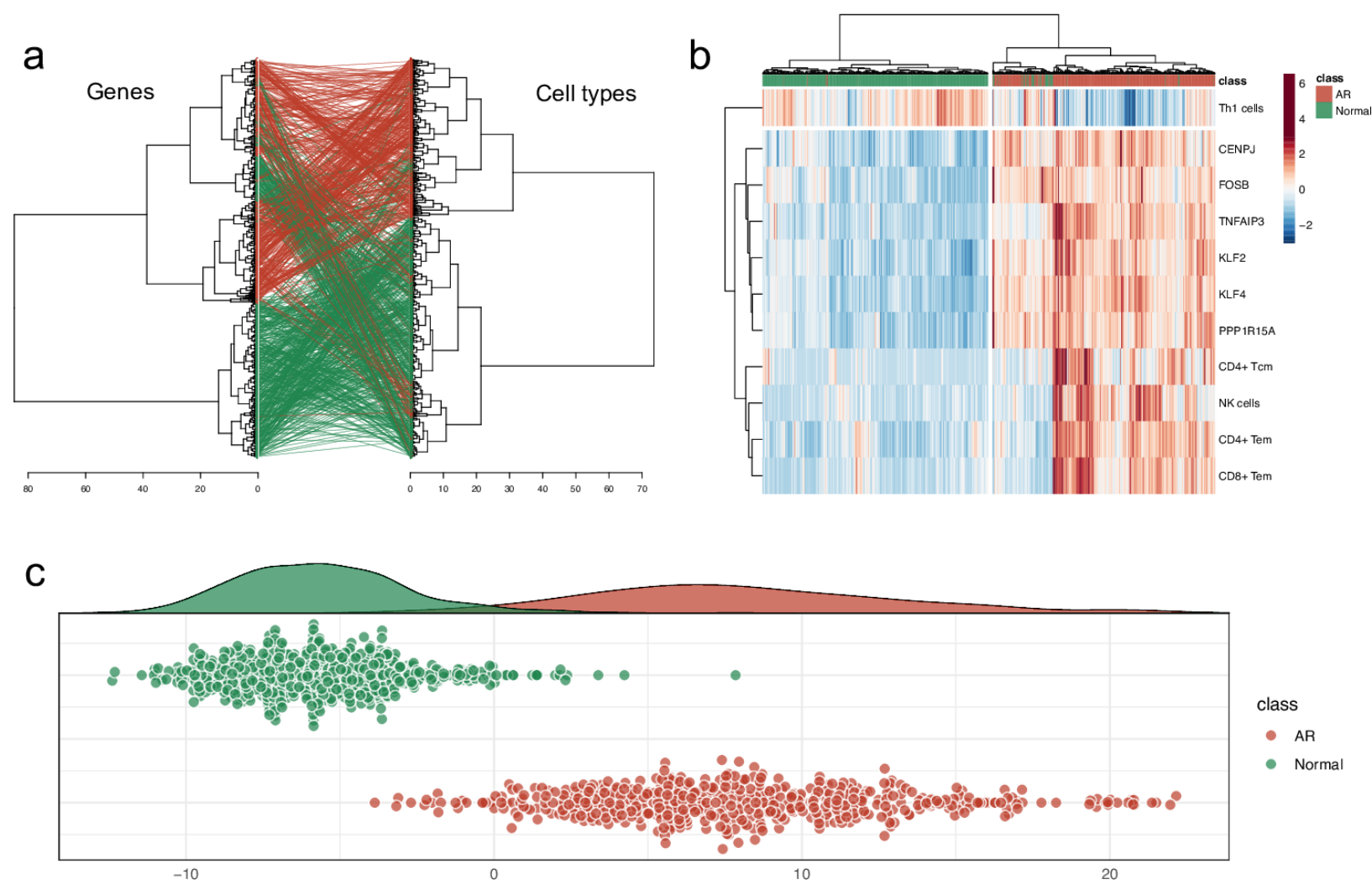

Fig 3. Feature selected genes and cell types and the Instability Score as their combination. (a) A comparison tanglegram between hierarchical clusterings based on Ward algorithm of AR and Normal samples for gene expression data with feature selected genes (left) and enrichment scores with feature selected cell types (right). The lines represent the tracking of each sample. Red color represents AR samples and green color represents Normal samples. (b) The heatmap plot of combined selected features with AR and Normal samples. (c) Instability Score plot for AR and Normal samples.

\section{Leveraging Selected Features to Create a Scoring Function to Carry out Precision Sub- phenotyping of Stable Samples.}

Having generated a powerful and robust tool of in-silico sample classification by the InstaScore (Methods), we were able to apply this tool to the 1,154 transplant samples that were identified by pathologists/investigators in each of the datasets as histologically stable. With this approach, we are able recognize stable samples as more similar to Normal kidneys or as more similar to the rejected kidney allograft group, i.e. they would be molecularly stable (mSTA) or molecularly Acute Rejection (mAR). For this split of histologically stable transplants, we introduced new notations to reflect the deeper molecular sub-typing of functioning allografts: $h S T A / m S T A$ for molecular and histological evidence of no rejection or tissue injury, and $h S T A / m A R$ for allografts with clearer evidence of ongoing molecular rejection. The InstaScore identified $46 \%$ of all hSTA 
grafts $(n=528)$ as having mAR ( $\underline{\text { Fig } 4 a)}$. This "misclassification rate" of pathology is in a line with the previously reported discrepancies in transplant phenotyping across different pathologists $[13,14]$.
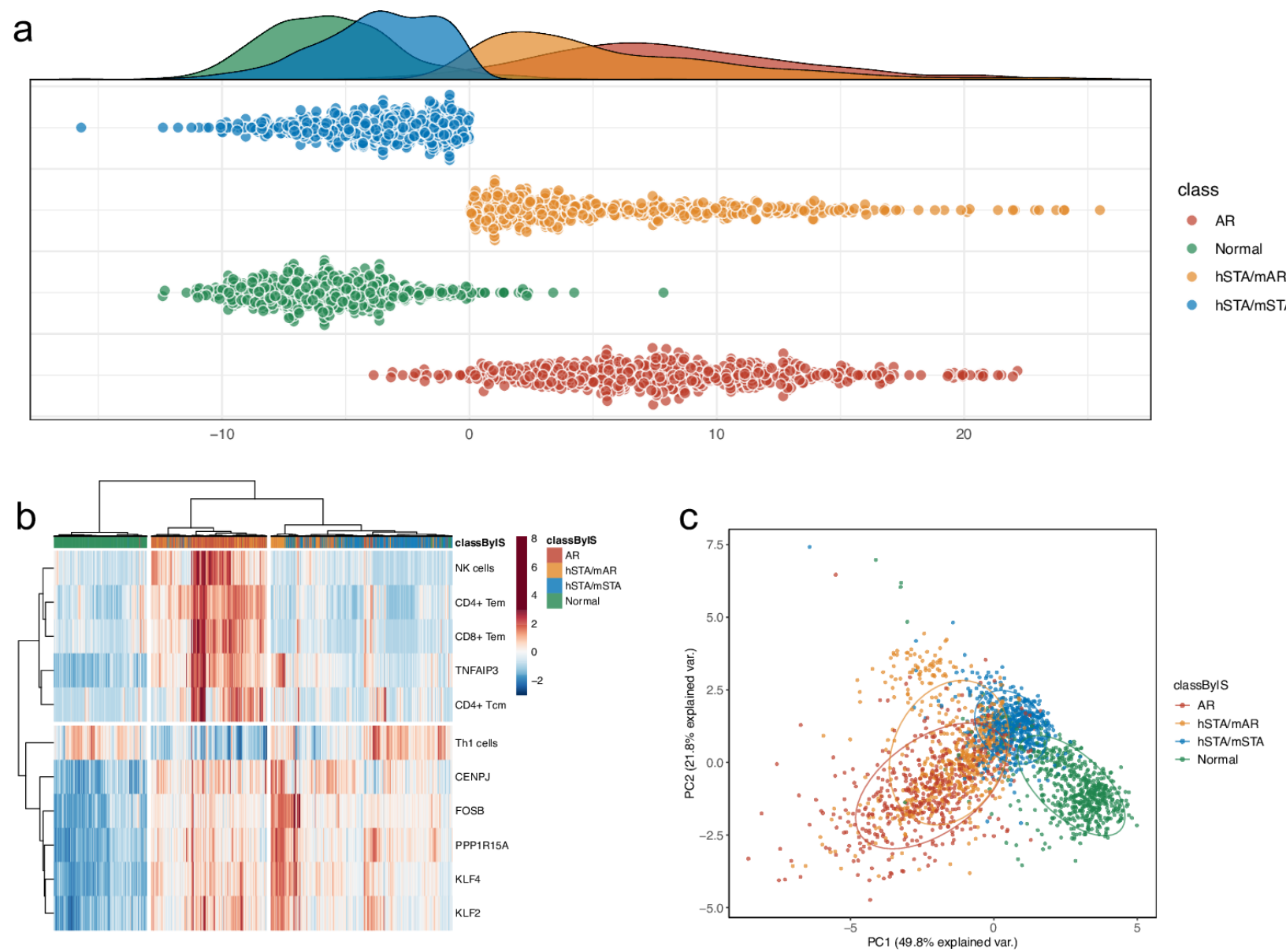

Fig 4. Plots of AR, sub-phenotyped hSTA, and Normal samples based on InstaScore results. (a) The Instability Score plots for AR, Normal, hSTA/mAR, and hSTA/mSTA samples. (b) Heatmap and (c) PCA plots of AR and Normal samples with sub-phenotyped hSTA samples based on the InstaScore.

We represented the scores for each sample in the form of the scatter plot (Figs 3c, 4a). The InstaScore can significantly distinguish AR and Normal samples ( $p=7 e-179 ;$ Fig $3 c$ ), giving the ability to identify hSTA/mAR and hSTA/mSTA samples ( $p=1 \mathrm{e}-188$; Fig 4a) by thresholding with 0 . From visualizing all the data with the selected features in the form of the heatmap (Fig $4 b$ ) and PCA plot (Fig 4c) and coloring sub-phenotypes based on the InstaScore one can notice that $\mathrm{hSTA} / \mathrm{mAR}$ samples clustered nearly perfectly with AR samples separately from hSTA/mSTA samples and could be seen as ones in the intermediate stage between Normal and AR samples.

Validation of hSTA sub-phenotyping using clinical follow-up data. In order to further demonstrate the functional relevance of the InstaScore by gene expression and cell types, we explored the clinical utility of the InstaScore in an independent microarray data set from 67 unique STA patients (stable clinical graft function, no DSA, no AR) from a randomized controlled 
clinical trial (NCT00141037) with transcriptional data on serial protocol kidney transplant biopsies at 0, 3, 6, 12 , and 24 months $[59,60]$ and with longitudinal functional outcomes up to 5 years after initial engraftment. We had the unique opportunity to test the impact of the locked InstaScore on the change of estimated glomerular filtration rate (eGFR) and graft loss events over this time period. We correlated the InstaScore values with those outcomes and found higher correlation values for cell type infiltration/activation model with delta eGFR ( Fig 5a) $r=$ $0.52(p=6.4 e-6)$ and graft loss events $r=0.17$.
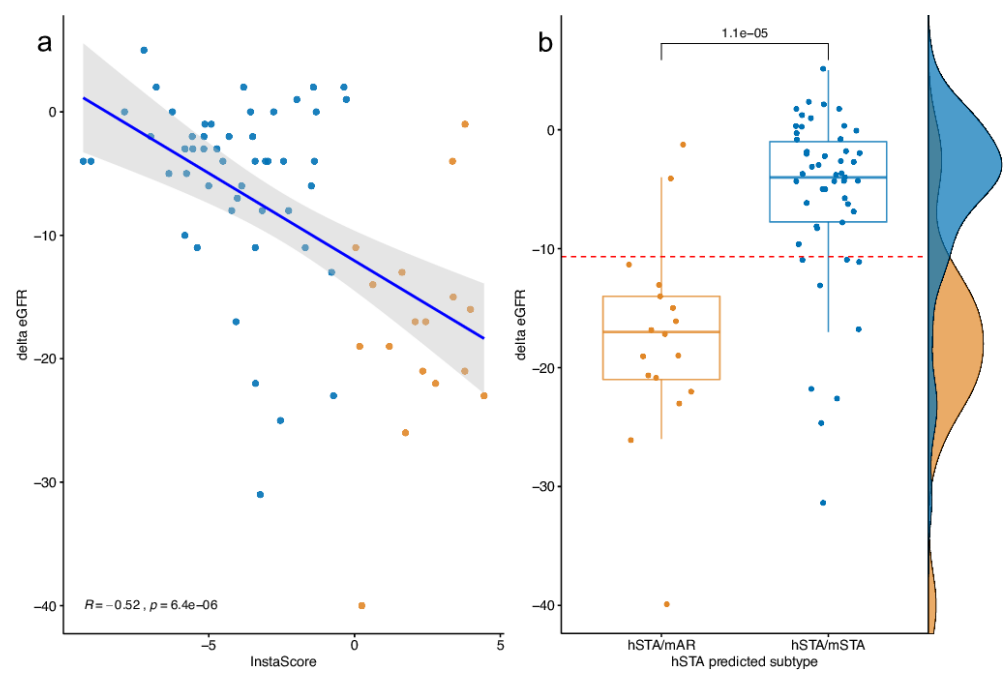

Fig 5. Validation plots on the independent clinical data.

(a) Correlation plot between change in eGFR over 1-5 year period after biopsy and the InstaScore (cor $=-0.52, p=6.4 \mathrm{e}-6$ ). (b) Boxplot of delta eGFR distributions for predicted hSTA subpopulations by the InstaScore $(p=1.1 \mathrm{e}-5)$.

Based on a separation of the hSTA groups by the InstaScore, the predicted hSTA subphenotypes, showed a significant separation over the 5-year follow-up with a delta eGFR separation of greater than -10 . Fig 5b represents boxplot comparisons of InstaScores between predicted sub-phenotypes with this delta eGFR thresholding of $>-10$ and shows that the groups difference was significant $(p=1.1 e-5)$. Therefore, we found that the InstaScore on the 6 month protocol biopsy can be valuable for predicting graft function outcomes 5 years later and thus can help differentiate those patients who are more likely to have progressive graft injury and decline in graft function over time, even though the 6 month protocol biopsy or DSAs at the 6 month time point post-transplant would not have been able to provide the same predictive discriminatory power. 


\section{Discussion}

In this study we identified discrepancies in the current histological recognition of stable allografts and demonstrated a new Instability Score (or InstaScore), a linear combination of selected features based on the input from tissue gene expression and inferred cell type biopsy molecular data modeled on AR biology, that provides precise (sub-)phenotyping and early recognition of molecular and cellular rejection of otherwise functionally and histologically stable allografts on protocol biopsies. Unlike other published studies by others [61-65] and our group $[2,54,66,67]$ that have only studied gene expression transcriptional perturbation in AR, this is the first development and application of a combined genes and cell types into Instability Score in tissue that can rapidly reclassify samples into molecularly and even more importantly, functionally relevant, diverse groups: those most similar to the normal kidneys (hSTA/mSTA) and those most similar to the rejected kidneys (hSTA/mAR).

We employed machine learning methods to better understand the underlying mechanisms in stable kidney transplants and show possible reasons for discrepancy in histological diagnostics. By taking advantage of the statistical power of a large collective gene expression dataset and adopting a novel computational tool of cell type enrichment analysis, xCell [51], we refined the molecular and cellular profile of AR to narrow the signature to specific genes and cell types that precisely map rejection, and then used the logistic regression algorithm to build a linear combination of features, the InstaScore, to identify two major subgroups of samples, otherwise described homogenously as stable in the public datasets. About half (54\%) of the histologically defined STA (hSTA) had immune quiescence and molecular similarities to the healthy donor kidney, and have been redefined in this study as histologically and molecularly stable (hSTA/mSTA), a large number (46\%) of the remaining biopsies had molecular evidence of rejection (hSTA/mAR) and should not have been classified as stable allografts. The distribution of the hSTA/mAR stable biopsies were found to be scattered across multiple datasets, supporting that their presence is not due to failure of histological characterization at any particular transplant program. Independent validation of the InstaScore was conducted on microarray data from a set of biopsies not utilized for model training and identified subgroups of hSTA/mAR phenotypes again on 6 months protocol biopsies, from otherwise presumed functionally and histologically stable transplant recipients. The InstaScore application on these biopsies, where long term outcome data was available, stratified these kidney transplant recipients as being at greater risk of chronic graft rejection, more progressive interstitial fibrosis and tubular atrophy and thus, greater loss of graft function over subsequent follow-up. This critical information, if available to the physician in real time, can allow for recipient immunosuppression stratification, aggressive clinical follow-up and graft salvage; all of which are critical unmet needs in transplantation.

The 6 feature selected genes, KLF4, CENPJ, KLF2, PPP1R15A, FOSB, and TNFAIP3, contributed into the InstaScore, are significantly dysregulated and were highly associated with AR in comparison to normal kidneys. As expected, these significantly upregulated genes are enriched in the regulation of the immune response, cell aggregation and activation, and innate immunity. KLF2, KLF4, and TNFAIP3 belong to zinc finger superfamily, where KLF 4 and 2, both belongs to the same group of Kruppel (zinc-finger) family of transcription factors and are known 
to regulate kidney injury/disease [68]. KLF2 is vasoprotective and KLF4 is renoprotective, both are enriched in the endothelium and have overlapping functions and similar downstream targets [69-71]. In AR, increased expression of $K L F 4$ and 2 is also associated with endothelial ischemia reperfusion injury [72]. Zinc finger protein TNFAIP3 has anti-apoptotic and antiinflammatory functions and its expression localizes mainly to endothelial cells and infiltrating myeloid cells and expression of TNFAIP3 by infiltrating T cells (myeloid cells) results in adverse clinical outcomes[73-75]. CENPJ functions as a transcriptional coactivator in the STAT5 signaling pathway, and TNF-induced NF-kappaB-mediated transcription [76,77], both central regulators of inflammation. Phosphatase PPP1R15A is only expressed in stressed cells and negatively regulate acute kidney injury vis Type 1 interferon (IFN). Its overexpression has been observed in chronic inflammatory diseases such as lupus, which also has a type I IFN signature [78]. Type 1 IFN has a direct effect on CD8+ T cells, promoting clonal expansion and memory T-cell, plasma cell differentiation, and enhance B-cell responses [79]. FOSB expression has been associated with the progression of renal disease including IgA nephropathy, leading to end-stage renal failure [80]. Thus the discovery gene signatures from kidney biopsies in the InstaScore are crucial for - endothelial cell integrity, T-cell expansion and activity, and have been previously associated with inferior graft function and rejection [81,82]. Importantly, the utility of this gene set in the InstaScore prediction is significant, so application of a rapid PCR based assay on formalin fixed paraffin embedded slices from the pathology samples, could allow for triaging the molecular burden of injury on a protocol biopsy, without the need to obtain an additional "research" core for snap freezing or RNAlater, is generally recommended by previous studies. This approach has been developed and published recently by our group $[83,84]$.

The 5 feature selected cell types are also in line with organ rejection biology[85]. AR is associated with early infiltration of CD8+ T and CD4+ T cells, macrophages, NK cells, and B cells $[86,87]$. Transplanted kidneys lose their function due to both immunologic and metabolic mechanisms but the most predominant cause of graft loss, according to the latest Banff classification of renal allograft pathology, is alloimmune response with the major role of T-cell in acute rejection [88]. From our feature selection procedure, we identified 5 cell types from the original set of 64 cell types (from which 37 were enriched in AR): CD4+ Tcm, CD4+ Tem, CD8+ Tem, NK cells, and Th1 cells, with CD4+ Tcm having the largest effect size. NK cell are pathogenesis-linked markers for antibody mediated rejection [89] and are known to regulate diverse $T$ cell responses promoting T-cell proliferation and graft rejection [90].In the immunologic response to the allograft, $T$ cells terminally differentiate and divide into central memory T cells (Tcm), and effector memory T cells (Tem) [91]. Tem can be both CD8+ and $\mathrm{CD} 4+$, where both produce IFN-gamma, IL-4, and IL-5 within hours following antigenic stimulation, however, CD8+ Tem carry large amounts of cytotoxic molecules like granzyme, granulysin and perforin [92,93]. In our feature selection procedure, we identified both, CD4+ Tem, CD8+ Tem, as the two enriched cell types for AR, but the classification model recognized CD4+ Tcm having the largest effect size in this model. This is likely because T central memory cells mediate reactive memory, which has higher sensitivity to antigenic stimulation, is less dependent on co-stimulation, and provides more effective stimulatory feedback to dendritic cells (DC) and B cells. Compared to Tem, Tcm are characterized by slow effector function, with expansion and differentiation of T cells in response to repeat antigenic stimulation [94]. Thus the higher enrichment of CD4+ Tcm in the InstaScore may suggest the inherent, pre-determined 
ability of a hSTA/mAR graft, in the absence of substantive active inflammation at the time of biopsy, to be primed to differentiate into Tem even with lower levels of subsequent antigen recognition, such as with varying exposure to immunosuppression[95]. Thus, the InstaScore may be identifying a primed hSTA graft that is greater risk to subsequent immune injury and allograft damage as it already carries a primed signature of $m A R$, than an allograft that is classically stable (hSTA/mSTA).

Conversely, Th1 cells are less enriched in the InstaScore, which is a little surprising as Th1-cell subsets are pro-inflammatory, the Th2-cell subsets anti-inflammatory, and Th1-Th2 balance is regarded as the major mechanism of rejection [96]. The ratio Th1/Th2 in the InstaScore was decreased in comparison to Normal healthy control samples by 4.6 times, although an increase in the ratio is associated with AR as observed before $[97,98]$. We believe this difference may relate to the temporal nature of the TH1/Th2 state and a skew towards more $\mathrm{M} 2$ macrophages rather than M1 macrophages, as we are really detecting sub-clinical molecular rejection, rather than full-blown, clinically relevant $A R$, where the reverse findings are usually observed. Increased numbers of M2 than M1 macrophages have been found in some studies to correlate with graft-loss [99]. KLF4 expression in increased in the InstaScore, and interestingly this gene plays a key role to polarize macrophage into the $M 2$ subset [100], which undertake host defense and wound healing/tissue remodeling tasks[101] and thus can be found to be enriched in renal fibrosis [102].In the AR bopsies in this study, as expected, M1 macrophage signatures were higher in AR [103]. Thus, the macrophage subsets show a dynamic state of skewing the preponderance of $\mathrm{M} 2$ to $\mathrm{M} 1$ macrophages in the process of immunological priming from pre$A R$ and full blown AR, and the InstaScore uncovers the state of AR priming or pre-AR in histologically and clinically stable kidney transplants, further highlighting the value of identifying hSTA grafts that are thus at greater risk of AR over time.

Given the design of the study, there are a few inherent limitations. First, this study was based on publicly available data with limited access to clinical and demographic reports in each of the public data. We excluded 5 datasets and 12 samples from the study as they lacked any detailed annotations. Secondly, we found 257 duplicates where some had slightly different histological annotations based on study aims, thus we had to carefully filter samples to minimize errors in sample identification. Thirdly, as the gene expressions in some datasets used in the study were measured using much older technologies, we excluded two sets of data from the current analysis. The presence of batch effects was recognized issue in this kind of analysis and can be challenging to control for as it can contribute to false positive findings in meta-analysis studies, but we believe that the testing of multiple normalizing methods provides a robust methodology for greater confidence in the data.

In conclusion, we have generated an Instability Score for histologically and clinically stable allograft profiled by microarray or RNASeq technologies (InstaScore) - the linear combination of feature selected 6 genes and 5 cell type. We have demonstrated that when we apply this Score to 1,154 transplant samples that were identified by pathologists as hSTA samples it segregates hSTA samples, that would otherwise be grouped into a single bucket, now into quite different 
molecular subtypes, that were subsequently confirmed by independent data analysis to be very functionally diverse over time. Thus, thus is the first study that highlights that even among histologically stable kidney transplants there is wide heterogeneity that has to be addressed in transplant diagnostics and analysis. There is a need of a better and more precise method that can sub-phenotype stable allografts into those that are stable at all biological levels and those that are slowly sliding towards rejection. Based on the application of the InstaScore for transcriptional data, immunosuppression adjustments could be made in patients to preserve allograft function. We uncovered that accepting all samples classified as "stable" from different investigators and datasets will neglect the important biological diversity that naturally exists in the histological definition of a "stable" sample and will necessarily lead to false positive or false negative discoveries when attempting to study and fully understand allograft rejection. 
bioRxiv preprint doi: https://doi.org/10.1101/2019.1218 881706; this version posted December 19, 2019. The copyright holder for this preprint

(which was not certified by peer review) is the author/funder, who has granted bioRxiv a license to display the preprint in perpetuity. It is made available under aCC-BY-ND 4.0 International license.

\section{Acknowledgments}

We would like to thank Tara Sidgel, Jul Liberto, and Parhom Towfighi for collecting and organizing clinical data, to Silvia Pineda and Jieming Chen for fruitful discussions and suggestions, and all members of the SarwalLab and SirotaLab who supported all study efforts. We are also thankful to patients and their families whose deidentified samples have provided the needed data to make these study findings. 


\section{References}

1. Joshee P, Wood AG, Wood ER, Grunfeld EA. Meta-analysis of cognitive functioning in patients following kidney transplantation. Nephrol Dial Transplant. 2017; 1-10. doi:10.1093/ndt/gfx240

2. Naesens M, Khatri P, Li L, Sigdel TK, Vitalone MJ, Chen R, et al. Progressive histological damage in renal allografts is associated with expression of innate and adaptive immunity genes. Kidney Int. 2011;80: 1364-1376. doi:10.1038/ki.2011.245

3. Sigdel TK, Li L, Tran TQ, Khatri P, Naesens M, Sansanwal P, et al. Non-HLA antibodies to immunogenic epitopes predict the evolution of chronic renal allograft injury. J Am Soc Nephrol. 2012;23: 750-763. doi:10.1681/ASN.2011060596

4. Gulshan V, Peng L, Coram M, Stumpe MC, Wu D, Narayanaswamy A, et al. Development and validation of a deep learning algorithm for detection of diabetic retinopathy in retinal fundus photographs. JAMA - J Am Med Assoc. 2016;316: 2402-2410. doi:10.1001/jama.2016.17216

5. Nasr M, Sigdel T, Sarwal M. Advances in diagnostics for transplant rejection. Expert Rev Mol Diagn. Taylor \& Francis; 2016;16: 1121-1132. doi:10.1080/14737159.2016.1239530

6. Perkins D, Verma M, Park KJ. Advances of genomic science and systems biology in renal transplantation: A review. Semin Immunopathol. 2011;33: 211-218. doi:10.1007/s00281011-0243-2

7. Sirota M, Sarwal M. Transplantomics: Towards Precision Medicine in Transplantation Research. Transplantation. 2017; 1. doi:10.1097/TP.0000000000001664

8. Barrett T, Wilhite SE, Ledoux P, Evangelista C, Kim IF, Tomashevsky M, et al. NCBI GEO: Archive for functional genomics data sets - Update. Nucleic Acids Res. 2013;41: 991-995. doi:10.1093/nar/gks1193

9. Khatri P, Roedder S, Kimura N, De Vusser K, Morgan AA, Gong Y, et al. A common rejection module (CRM) for acute rejection across multiple organs identifies novel therapeutics for organ transplantation. J Exp Med. 2013;210: 2205-2221. doi:10.1084/jem.20122709

10. Choi J-W, Kim Y-H, Oh JW. Comparative Analyses of Signature Genes in Acute Rejection and Operational Tolerance. Immune Netw. 2017;17: 237. doi:10.4110/in.2017.17.4.237

11. Li L, Greene I, Readhead B, Menon MC, Kidd BA, Uzilov A V, et al. Novel Therapeutics Identification for Fibrosis in Renal Allograft Using Integrative Informatics Approach. Nat Publ Gr. 2016; 1-14. doi:10.1038/srep39487

12. Baron D, Ramstein G, Chesneau M, Echasseriau Y, Pallier A, Paul C, et al. A common gene signature across multiple studies relate biomarkers and functional regulation in tolerance to renal allograft. Kidney Int. 2015;87: 984-995. doi:10.1038/ki.2014.395

13. Furness PN, Taub N. International variation in the interpretation of renal transplant biopsies: Report of the CERTPAP project. Kidney Int. Elsevier Masson SAS; 2001;60: 1998-2012. doi:10.1046/j.1523-1755.2001.00030.x

14. Furness PN, Taub N, Assmann KJM, Banfi G, Cosyns JP, Dorman AM, et al. International variation in histologic grading is large, and persistent feedback does not improve reproducibility. Am J Surg Pathol. 2003;27: 805-810. doi:10.1097/00000478-20030600000012

15. Haas M, Loupy A, Lefaucheur C, Roufosse C, Glotz D, Seron D, et al. The Banff 2017 
Kidney Meeting Report: Revised diagnostic criteria for chronic active T cell-mediated rejection, antibody-mediated rejection, and prospects for integrative endpoints for nextgeneration clinical trials. Am J Transplant. 2018;18: 293-307. doi:10.1111/ajt.14625

16. Mengel $M$, Campbell $P$, Gebel $H$, Randhawa $P$, Rodriguez ER, Colvin R, et al. Precision diagnostics in transplantation: From bench to bedside. Am J Transplant. 2013;13: 562568. doi:10.1111/j.1600-6143.2012.04344.x

17. Halloran PF, Famulski KS, Reeve J. Molecular assessment of disease states in kidney transplant biopsy samples. Nat Rev Nephrol. Nature Publishing Group; 2016;12: 534-48. doi:10.1038/nrneph.2016.85

18. Reeve J, Böhmig GA, Eskandary F, Einecke G, Lefaucheur C, Loupy A, et al. Assessing rejection-related disease in kidney transplant biopsies based on archetypal analysis of molecular phenotypes. JCI insight. 2017;2: 1-14. doi:10.1172/jci.insight.94197

19. Reeve J, Sellarés J, Mengel M, Sis B, Skene A, Hidalgo L, et al. Molecular diagnosis of T cell-mediated rejection in human kidney transplant biopsies. Am J Transplant. 2013;13: 645-655. doi:10.1111/ajt.12079

20. Sellarés J, Reeve J, Loupy A, Mengel M, Sis B, Skene A, et al. Molecular diagnosis of antibody-mediated rejection in human kidney transplants. Am J Transplant. 2013;13: 971-983. doi:10.1111/ajt.12150

21. Halloran PF, Famulski KS, Chang J. A Probabilistic Approach to Histologic Diagnosis of Antibody-Mediated Rejection in Kidney Transplant Biopsies. Am J Transplant. 2017;17: 129-139. doi:10.1111/ajt.13934

22. Cosio FG, Grande JP, Wadei H, Larson TS, Griffin MD, Stegall MD. Predicting subsequent decline in kidney allograft function from early surveillance biopsies. Am J Transplant. 2005;5: 2464-2472. doi:10.1111/j.1600-6143.2005.01050.x

23. Waldron L, Riester M, Ramos M, Parmigiani G, Birrer M. The doppelganger effect: Hidden duplicates in databases of transcriptome profiles. J Natl Cancer Inst. 2016;108: 2-5. doi:10.1093/jnci/djw146

24. R core team. R: A language and environment for statistical computing. [Internet]. R Foundation for Statistical Computing, Vienna, Austria. Vienna, Austria: R Foundation for Statistical Computing; 2018. Available: http://www.r-project.org/

25. Piccolo SR, Sun Y, Campbell JD, Lenburg ME, Bild AH, Johnson WE. A single-sample microarray normalization method to facilitate personalized-medicine workflows. Genomics. Elsevier Inc.; 2012;100: 337-344. doi:10.1016/j.ygeno.2012.08.003

26. Huber W, Carey VJ, Gentleman R, Anders S, Carlson M, Carvalho BS, et al. Orchestrating high-throughput genomic analysis with Bioconductor. Nat Methods. Nature Publishing Group; 2015;12: 115-121. doi:10.1038/nmeth.3252

27. Irizarry RA. Exploration, normalization, and summaries of high density oligonucleotide array probe level data. Biostatistics. 2003;4: 249-264. doi:10.1093/biostatistics/4.2.249

28. Dai $M$, Wang $P$, Boyd AD, Kostov $G$, Athey $B$, Jones EG, et al. Evolving gene/transcript definitions significantly alter the interpretation of GeneChip data. Nucleic Acids Res. 2005;33: e175-e175. doi:10.1093/nar/gni179

29. Ritchie ME, Phipson B, Wu D, Hu Y, Law CW, Shi W, et al. limma powers differential expression analyses for RNA-sequencing and microarray studies. Nucleic Acids Res. 2015;43: e47-e47. doi:10.1093/nar/gkv007

30. Shi W, Oshlack A, Smyth GK. Optimizing the noise versus bias trade-off for Illumina whole 
genome expression BeadChips. Nucleic Acids Res. 2010;38. doi:10.1093/nar/gkq871

31. Durinck S, Spellman PT, Birney E, Huber W. Mapping identifiers for the integration of genomic datasets with the R/ Bioconductor package biomaRt. Nat Protoc. 2009;4: 11841191. doi:10.1038/nprot.2009.97

32. Leek JT, Scharpf RB, Bravo HC, Simcha D, Langmead B, Johnson WE, et al. Tackling the widespread and critical impact of batch effects in high-throughput data. Nat Rev Genet. Nature Publishing Group; 2010;11: 733-739. doi:10.1038/nrg2825

33. Jaffe AE, Hyde T, Kleinman J, Weinbergern DR, Chenoweth JG, McKay RD, et al. Practical impacts of genomic data "cleaning" on biological discovery using surrogate variable analysis. BMC Bioinformatics. BMC Bioinformatics; 2015;16: 372. doi:10.1186/s12859015-0808-5

34. Goh WW Bin, Wang W, Wong L. Why Batch Effects Matter in Omics Data, and How to Avoid Them. Trends Biotechnol. 2017;35: 498-507. doi:10.1016/j.tibtech.2017.02.012

35. Lazar C, Meganck S, Taminau J, Steenhoff D, Coletta A, Molter C, et al. Batch effect removal methods for microarray gene expression data integration: a survey. Brief Bioinform. 2013;14: 469-490. doi:10.1093/bib/bbs037

36. Nygaard V, Rødland EA, Hovig E. Methods that remove batch effects while retaining group differences may lead to exaggerated confidence in downstream analyses. Biostatistics. 2015;17: kxv027. doi:10.1093/biostatistics/kxv027

37. Müller C, Schillert A, Röthemeier C, Trégouët D-A, Proust C, Binder $H$, et al. Removing Batch Effects from Longitudinal Gene Expression - Quantile Normalization Plus ComBat as Best Approach for Microarray Transcriptome Data. PLoS One. 2016;11: e0156594. doi:10.1371/journal.pone.0156594

38. Shaham U, Stanton KP, Zhao J, Li H, Raddassi K, Montgomery R, et al. Removal of Batch Effects using Distribution-Matching Residual Networks. 2016; doi:10.1093/bioinformatics/btx196

39. Chen C, Grennan K, Badner J, Zhang D, Gershon E, Jin L, et al. Removing batch effects in analysis of expression microarray data: An evaluation of six batch adjustment methods. PLoS One. 2011;6. doi:10.1371/journal.pone.0017238

40. Johnson WE, Li C, Rabinovic A. Adjusting batch effects in microarray expression data using empirical Bayes methods. Biostatistics. 2007;8: 118-127. doi:10.1093/biostatistics/kxj037

41. Leek JT, Johnson WE, Parker HS, Jaffe AE, Storey JD. The SVA package for removing batch effects and other unwanted variation in high-throughput experiments. Bioinformatics. 2012;28: 882-883. doi:10.1093/bioinformatics/bts034

42. Bolstad BM, Irizarry RA, Astrand M, Speed TP. A comparison of normalization methods for high density oligonucleotide array data based on variance and bias. Bioinformatics. 2003;19: 185-193. doi:10.1093/bioinformatics/19.2.185

43. Gagnon-Bartsch JA, Speed TP. Using control genes to correct for unwanted variation in microarray data. Biostatistics. 2012;13: 539-552. doi:10.1093/biostatistics/kxr034

44. Oytam Y, Sobhanmanesh F, Duesing K, Bowden JC, Osmond-McLeod M, Ross J. Riskconscious correction of batch effects: Maximising information extraction from highthroughput genomic datasets. BMC Bioinformatics. BMC Bioinformatics; 2016;17: 1-17. doi:10.1186/s12859-016-1212-5

45. Tusher VG, Tibshirani R, Chu G. Significance analysis of microarrays applied to the 
ionizing radiation response. Proc Natl Acad Sci. 2001;98: 5116-5121.

doi:10.1073/pnas.091062498

46. Schwender H. Identifying differentially expressed genes with siggenes. : 1-30.

47. Goeman JJ, Solari A. Multiple hypothesis testing in genomics. Stat Med. 2014;33: 19461978. doi:10.1002/sim.6082

48. Reiner-Benaim A. FDR control by the BH procedure for two-sided correlated tests with implications to gene expression data analysis. Biometrical J. 2007;49: 107-126.

doi:10.1002/bimj.200510313

49. Yu G, Wang L-G, Han Y, He Q-Y. clusterProfiler: an R Package for Comparing Biological Themes Among Gene Clusters. Omi A J Integr Biol. 2012;16: 284-287.

doi:10.1089/omi.2011.0118

50. Szklarczyk D, Gable AL, Lyon D, Junge A, Wyder S, Huerta-Cepas J, et al. STRING v11: Protein-protein association networks with increased coverage, supporting functional discovery in genome-wide experimental datasets. Nucleic Acids Res. Oxford University Press; 2019;47: D607-D613. doi:10.1093/nar/gky1131

51. Aran D, Hu Z, Butte AJ. xCell: digitally portraying the tissue cellular heterogeneity landscape. Genome Biol. Genome Biology; 2017;18: 220. doi:10.1186/s13059-017-13491

52. Kuhn M. Building Predictive Models in R Using the caret Package. J Stat Softw. 2008;28: 159-160. doi:10.18637/jss.v028.i05

53. Perez-Riverol Y, Kuhn M, Vizcaíno JA, Hitz M-P, Audain E. Accurate and fast feature selection workflow for high-dimensional omics data. Zou Q, editor. PLoS One. 2017;12: e0189875. doi:10.1371/journal.pone.0189875

54. Sarwal M, Chua M-S, Kambham N, Hsieh S-C, Satterwhite T, Masek M, et al. Molecular Heterogeneity in Acute Renal Allograft Rejection Identified by DNA Microarray Profiling. N Engl J Med. 2003;349: 125-138. doi:10.1056/NEJMoa035588

55. Halloran PF, Venner JM, Madill-Thomsen KS, Einecke G, Parkes MD, Hidalgo LG, et al. Review: The transcripts associated with organ allograft rejection. Am J Transplant. 2018;18: 785-795. doi:10.1111/ajt.14600

56. Park WD, Stegall MD. A meta-analysis of kidney microarray datasets: Investigation of cytokine gene detection and correlation with rt-PCR and detection thresholds. BMC Genomics. 2007;8: 1-9. doi:10.1186/1471-2164-8-88

57. Stegall M, Park W, Kim D, Kremers W. Gene expression during acute allograft rejection: Novel statistical analysis of microarray data. Am J Transplant. 2002;2: 913-925. doi:10.1034/j.1600-6143.2002.21007.x

58. Szklarczyk D, Franceschini A, Wyder S, Forslund K, Heller D, Huerta-Cepas J, et al. STRING v10: protein-protein interaction networks, integrated over the tree of life. Nucleic Acids Res. 2015;43: D447-52. doi:10.1093/nar/gku1003

59. Sarwal MM, Ettenger RB, Dharnidharka V, Benfield M, Mathias R, Portale A, et al. Complete steroid avoidance is effective and safe in children with renal transplants: $A$ multicenter randomized trial with three-year follow-up. Am J Transplant. 2012;12: 27192729. doi:10.1111/j.1600-6143.2012.04145.x

60. Naesens M, Salvatierra O, Benfield M, Ettenger RB, Dharnidharka V, Harmon W, et al. Subclinical inflammation and chronic renal allograft injury in a randomized trial on steroid avoidance in pediatric kidney transplantation. Am J Transplant. 2012;12: 2730- 
2743. doi:10.1111/j.1600-6143.2012.04144.x

61. Cippà PE, Liu J, Sun B, Kumar S, Naesens M, McMahon AP. A late B lymphocyte action in dysfunctional tissue repair following kidney injury and transplantation. Nat Commun. Springer Nature; 2019;10: 1157. doi:10.1038/s41467-019-09092-2

62. Dorr CR, Oetting WS, Jacobson PA, Israni AK. Genetics of acute rejection after kidney transplantation. Transpl Int. Wiley; 2018;31: 263-277. doi:10.1111/tri.13084

63. Edemir B, Reuter S, Borgulya R, Schröter R, Neugebauer U, Gabriëls G, et al. Acute Rejection Modulates Gene Expression in the Collecting Duct. J Am Soc Nephrol. American Society of Nephrology (ASN); 2008;19: 538-546. doi:10.1681/asn.2007040513

64. Hernandez-Fuentes M, Christakoudi S, Runglall M, Mobillo P, Tsui T-L, Karma Y, et al. A Signature of Gene Expression in Peripheral Blood that Enables Earlier Detection of Acute Rejection in Kidney Transplant Recipients. Transplantation. Ovid Technologies (Wolters Kluwer Health); 2018;102: S180. doi:10.1097/01.tp.0000542822.75680.9c

65. Spivey TL, Uccellini L, Ascierto ML, Zoppoli G, De Giorgi V, Delogu LG, et al. Gene expression profiling in acute allograft rejection: Challenging the immunologic constant of rejection hypothesis. J Transl Med. Springer Nature; 2011;9: 174. doi:10.1186/14795876-9-174

66. Pineda S, Sigdel TK, Chen J, Jackson AM, Sirota M, Sarwal MM. Novel NonHistocompatibility Antigen Mismatched Variants Improve the Ability to Predict AntibodyMediated Rejection Risk in Kidney Transplant. Front Immunol. 2017;8: 1-17. doi:10.3389/fimmu.2017.01687

67. Sigdel TK, Bestard O, Tran TQ, Hsieh S-C, Roedder S, Damm I, et al. A Computational Gene Expression Score for Predicting Immune Injury in Renal Allografts. Câmara NOS, editor. PLoS One. 2015;10: e0138133. doi:10.1371/journal.pone.0138133

68. Rane MJ, Zhao Y, Cai L. Krüppel-like factors (KLFs) in renal physiology and disease. EBioMedicine. Elsevier BV; 2019;40: 743-750. doi:10.1016/j.ebiom.2019.01.021

69. Boon RA, Leyen TA, Fontijn RD, Fledderus JO, Baggen JMC, Volger OL, et al. KLF2-induced actin shear fibers control both alignment to flow and JNK signaling in vascular endothelium. Blood. American Society of Hematology; 2010;115: 2533-2542. doi:10.1182/blood-2009-06-228726

70. Ke B, Zhang A, Wu X, Fang X. The Role of Krüppel-like Factor 4 in Renal Fibrosis. Front Physiol. Frontiers Media SA; 2015;6. doi:10.3389/fphys.2015.00327

71. Sangwung P, Zhou G, Nayak L, Chan ER, Kumar S, Kang D-W, et al. KLF2 and KLF4 control endothelial identity and vascular integrity. JCI Insight. American Society for Clinical Investigation; 2017;2. doi:10.1172/jci.insight.91700

72. Mallipattu SK, Estrada CC, He JC. The critical role of Krüppel-like factors in kidney disease. Am J Physiol Physiol. American Physiological Society; 2016;312: F259-F265. doi:10.1152/ajprenal.00550.2016

73. Luft FC. Zinc fingers protect the kidney from ischemia/reperfusion injury. J Mol Med. Springer Science and Business Media LLC; 2008;86: 1297-1300. doi:10.1007/s00109-0080411-6

74. Lutz J, Luong LA, Strobl M, Deng M, Huang H, Anton M, et al. The A20 gene protects kidneys from ischaemia/reperfusion injury by suppressing pro-inflammatory activation. J Mol Med. Springer Science and Business Media LLC; 2008;86: 1329-1339.

doi:10.1007/s00109-008-0405-4 
75. Avihingsanon Y, Ma N, Csizmadia E, Wang C, Pavlakis M, Giraldo M, et al. Expression of protective genes in human renal allografts: A regulatory response to injury associated with graft rejection. Transplantation. Ovid Technologies (Wolters Kluwer Health); 2002;73: 1079-1085. doi:10.1097/00007890-200204150-00011

76. Koyanagi M, Hijikata M, Watashi K, Masui O, Shimotohno K. Centrosomal P4.1-associated protein is a new member of transcriptional coactivators for nuclear factor-kB. J Biol Chem. American Society for Biochemistry \& Molecular Biology (ASBMB); 2005;280: 12430-12437. doi:10.1074/jbc.M410420200

77. Peng B, Sutherland KD, Sum EYM, Olayioye M, Wittlin S, Tang TK, et al. CPAP Is a Novel Stat5-Interacting Cofactor that Augments Stat5-Mediated Transcriptional Activity. Mol Endocrinol. The Endocrine Society; 2002;16: 2019-2033. doi:10.1210/me.2002-0108

78. Plazy C, Dumestre-Pérard C, Sarrot-Reynauld F, Audoin P, Quesada JL, Pierre P, et al. Letter to the Editor: Protein phosphatase 1 subunit Ppp1r15a/GADD34 is overexpressed in systemic lupus erythematosus and related to the expression of type I interferon response genes. Autoimmun Rev. Elsevier BV; 2019;18: 211-213. doi:10.1016/j.autrev.2018.09.007

79. Kamphuis E, Junt T, Waibler Z, Forster R, Kalinke U. Type I interferons directly regulate lymphocyte recirculation and cause transient blood lymphopenia. Blood. American Society of Hematology; 2006;108: 3253-3261. doi:10.1182/blood-2006-06-027599

80. Park HJ, Kim JW, Cho BS, Chung JH. Association of FOS-Like Antigen 1 Promoter Polymorphism with Podocyte Foot Process Effacement in Immunoglobulin A Nephropathy Patients. J Clin Lab Anal. Wiley; 2014;28: 391-397. doi:10.1002/jcla.21699

81. Lin Y, Lewallen EA, Camilleri ET, Bonin CA, Jones DL, Dudakovic A, et al. RNA-seq analysis of clinical-grade osteochondral allografts reveals activation of early response genes. J Orthop Res. Wiley; 2016;34: 1950-1959. doi:10.1002/jor.23209

82. Mutoh J, Ohsawa $\mathrm{M}$, Hisa $\mathrm{H}$. Effect of naloxone on ischemic acute kidney injury in the mouse. Neuropharmacology. Elsevier BV; 2013;71: 10-18.

doi:10.1016/j.neuropharm.2013.03.001

83. Sigdel TK, Bestard O, Salomonis N, Hsieh S-C, Torras J, Naesens M, et al. Intragraft Antiviral-Specific Gene Expression as a Distinctive Transcriptional Signature for Studies in Polyomavirus-Associated Nephropathy. Transplantation. 2016;00: 1. doi:10.1097/TP.0000000000001214

84. Sigdel T, Nguyen M, Liberto J, Dobi D, Junger H, Vincenti F, et al. Assessment of 19 Genes and Validation of CRM Gene Panel for Quantitative Transcriptional Analysis of Molecular Rejection and Inflammation in Archival Kidney Transplant Biopsies. Front Med. 2019;6. doi:10.3389/fmed.2019.00213

85. Farrar CA, Kupiec-Weglinski JW, Sacks SH. The innate immune system and transplantation. Cold Spring Harb Perspect Med. Cold Spring Harbor Laboratory; 2013;3: a015479-a015479. doi:10.1101/cshperspect.a015479

86. Imig JD, Ryan MJ. Immune and inflammatory role in renal disease. Comprehensive Physiology. John Wiley \& Sons, Inc.; 2013. pp. 957-976. doi:10.1002/cphy.c120028

87. Weller S, Varrier M, Ostermann M. Lymphocyte Function in Human Acute Kidney Injury. Nephron. S. Karger AG; 2017;137: 287-293. doi:10.1159/000478538

88. Ingulli E. Mechanism of cellular rejection in transplantation. Pediatr Nephrol. Springer Nature; 2010;25: 61-74. doi:10.1007/s00467-008-1020-x 
89. Yazdani S, Callemeyn J, Gazut S, Lerut E, de Loor H, Wevers M, et al. Natural killer cell infiltration is discriminative for antibody-mediated rejection and predicts outcome after kidney transplantation. Kidney Int. Elsevier BV; 2019;95: 188-198.

doi:10.1016/j.kint.2018.08.027

90. Crome SQ, Lang PA, Lang KS, Ohashi PS. Natural killer cells regulate diverse T cell responses. Trends Immunol. Elsevier BV; 2013;34: 342-349. doi:10.1016/j.it.2013.03.002

91. Sallusto F, Geginat J, Lanzavecchia A. Central memory and effector memory T cell subsets: function, generation, and maintenance. Annu Rev Immunol. 2004;22: 745-63. doi:10.1146/annurev.immunol.22.012703.104702

92. Opata M, Stephens R. Early Decision: Effector and Effector Memory T Cell Differentiation in Chronic Infection. Curr Immunol Rev. Bentham Science Publishers Ltd.; 2014;9: 190206. doi:10.2174/1573395509666131126231209

93. Sarwal MM, Jani A, Chang S, Huie P, Wang Z, Salvatierra O, et al. Granulysin expression is a marker for acute rejection and steroid resistance in human renal transplantation. Hum Immunol. 2001;62: 21-31. doi:10.1016/S0198-8859(00)00228-7

94. Pepper M, Jenkins MK. Origins of CD4+ effector and central memory T cells. Nat Immunol. Springer Nature; 2011;12: 467-471. doi:10.1038/ni.2038

95. Segundo DS, Fernández-Fresnedo G, Gago M, Beares I, González M, Ruiz JC, et al. Changes in the number of circulating T CM and T em subsets in renal transplantation: Relationship with acute rejection and induction therapy. Kidney Int Suppl. Elsevier BV; 2011;1: 31-35. doi:10.1038/kisup.2011.9

96. Liu Z, Fan H, Jiang S. CD4+ T-cell subsets in transplantation. Immunol Rev. Wiley; 2013;252: 183-191. doi:10.1111/imr.12038

97. Karczewski J, Karczewski M, Glyda M, Wiktorowicz K. Role of TH1/TH2 Cytokines in Kidney Allograft Rejection. TPS. Elsevier Inc.; 2008;40: 3390-3392.

doi:10.1016/j.transproceed.2008.07.125

98. Illigens BM, Yamada A, Anosova N, Dong VM, Sayegh MH, Benichou G. Dual effects of the alloresponse by Th1 and Th2 cells on acute and chronic rejection of allotransplants. Eur J Immunol. Wiley; 2009;39: 3000-3009. doi:10.1002/eji.200838980

99. Martinez FO, Gordon S. The M1 and M2 paradigm of macrophage activation: time for reassessment. F1000Prime Rep. F1000 ( Faculty of 1000 Ltd); 2014;6. doi:10.12703/p6-13

100. Wang K, Zhou W, Cai Q, Cheng J, Cai R, Xing R. SUMOylation of KLF4 promotes IL-4 induced macrophage M2 polarization. Cell Cycle. Informa UK Limited; 2017;16: 374-381. doi:10.1080/15384101.2016.1269045

101. Roszer T. Understanding the mysterious $M 2$ macrophage through activation markers and effector mechanisms. Mediators Inflamm. Hindawi Limited; 2015;2015: 1-16. doi:10.1155/2015/816460

102. Chen T, Cao Q, Wang Y, Harris DCH. M2 macrophages in kidney disease: biology, therapies, and perspectives. Kidney Int. Elsevier BV; 2019;95: 760-773. doi:10.1016/j.kint.2018.10.041

103. Huang G, Wilson NA, Reese SR, Jacobson LM, Zhong W, Djamali A. Characterization of transfusion-elicited acute antibody-mediated rejection in a rat model of kidney transplantation. Am J Transplant. Wiley; 2014;14: 1061-1072. doi:10.1111/ajt.12674

104. Eisenberg E, Levanon EY. Human housekeeping genes, revisited. Trends Genet. Elsevier Ltd; 2013;29: 569-574. doi:10.1016/j.tig.2013.05.010 
105. Zhang Y, Li D, Sun B. Do housekeeping genes exist? PLoS One. 2015;10: 1-22. doi:10.1371/journal.pone.0123691

106. Venet $D$, Dumont JE, Detours V. Most random gene expression signatures are significantly associated with breast cancer outcome. PLoS Comput Biol. 2011;7. doi:10.1371/journal.pcbi.1002240

107. Freytag S, Gagnon-Bartsch J, Speed TP, Bahlo M. Systematic noise degrades gene coexpression signals but can be corrected. BMC Bioinformatics. BMC Bioinformatics; 2015;16: 309. doi:10.1186/s12859-015-0745-3

108. Jacob L. RUV for normalization of expression array data. 2015; 1-16.

109. Risso D, Ngai J, Speed TP, Dudoit S. Normalization of RNA-seq data using factor analysis of control genes or samples. Nat Biotechnol. 2014;32: 896-902. doi:10.1038/nbt.2931

110. Reese SE, Archer KJ, Therneau TM, Atkinson EJ, Vachon CM, de Andrade M, et al. A new statistic for identifying batch effects in high-throughput genomic data that uses guided principal component analysis. Bioinformatics. 2013;29: 2877-2883.

doi:10.1093/bioinformatics/btt480 


\section{Manuscript Figures:}

\section{Fig 1. Flow chart}

(a) Data pre-processing and Normalization. (b) Feature selection and creating the Instability Score (InstaScore). (c) Applying InstaScore to hSTA for sub-phenotyping and validating results

Fig 2. The heatmap plots for differentially expressed genes and significantly enriched cell types.

(a) Heatmap clustering plot for significant genes from SAM analysis of AR vs Normals. (b) Heatmap clustering plots for significant cell types from the non-parametric Wilcoxon statistical test $(\mathrm{BH}$ p-adj $<0.05)$ in the analysis of AR vs Normals.

Fig 3. Feature selected genes and cell types and the Instability Score as their combination. (a) A comparison tanglegram between hierarchical clusterings based on Ward algorithm of AR and Normal samples for gene expression data with feature selected genes (left) and enrichment scores with feature selected cell types (right). The lines represent the tracking of each sample. Red color represents AR samples and green color represents Normal samples. (b) The heatmap plot of combined selected features with AR and Normal samples. (c) Instability Score plot for AR and Normal samples.

Fig 4. Plots of AR, sub-phenotyped hSTA, and Normal samples based on InstaScore results. (a) The Instability Score plots for AR, Normal, hSTA/mAR, and hSTA/mSTA samples. (b) Heatmap and (c) PCA plots of AR and Normal samples with sub-phenotyped hSTA samples based on the InstaScore.

Fig 5. Validation plots on the independent clinical data.

(a) Correlation plot between change in eGFR over 1-5 year period after biopsy and the InstaScore (cor $=-0.52, p=6.4 e-6$ ). (b) Boxplot of delta eGFR distributions for predicted hSTA subpopulations by the InstaScore $(p=1.1 \mathrm{e}-5)$. 
bioRxiv preprint doi: https://doi.org/10.1101/2019.12.18.881706; this version posted December 19,2019 . The copyright holder for this preprint (which was not certified by peer review) is the author/funder, who has granted bioRxiv a license to display the preprint in perpetuity. It is made available under aCC-BY-ND 4.0 International license.

\section{Supplementary Figures:}
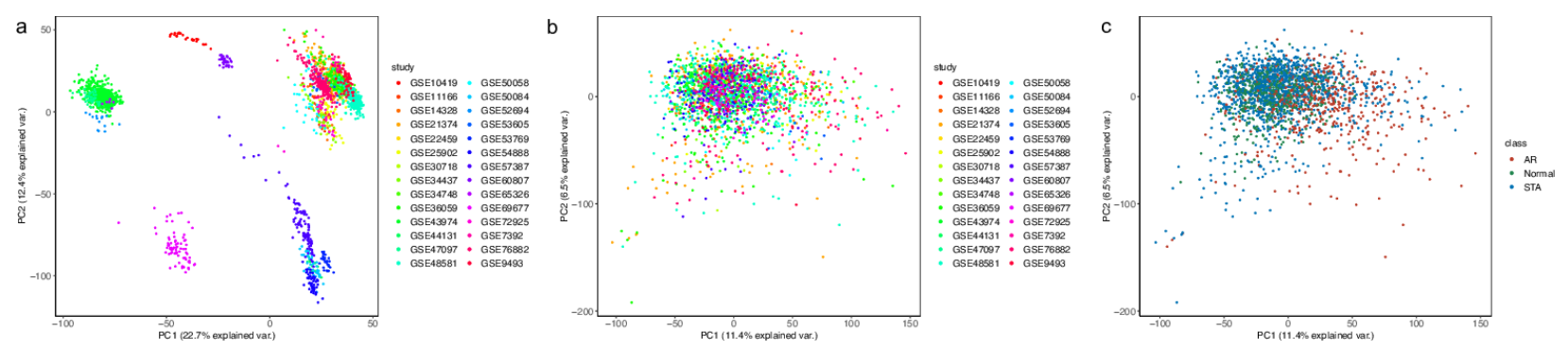

Fig S1. Scatter plots of first two principal components of gene expression data.

PCA plots (a) before normalization colored by study, (b) after normalization with ComBat colored by study, (c) after normalization colored by phenotypes.

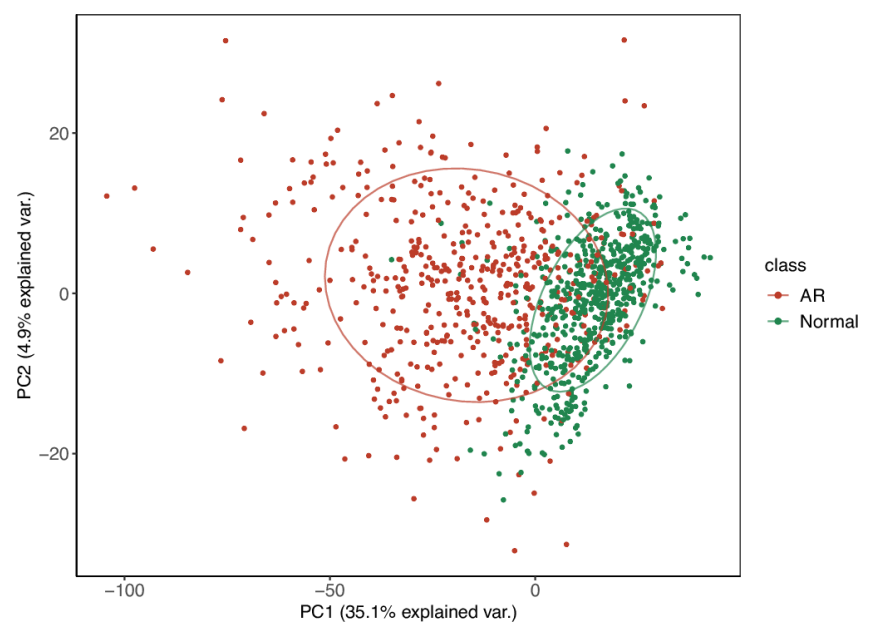

Fig S2. PCA clustering plot for differentially expressed genes from analysis of AR vs Normals 

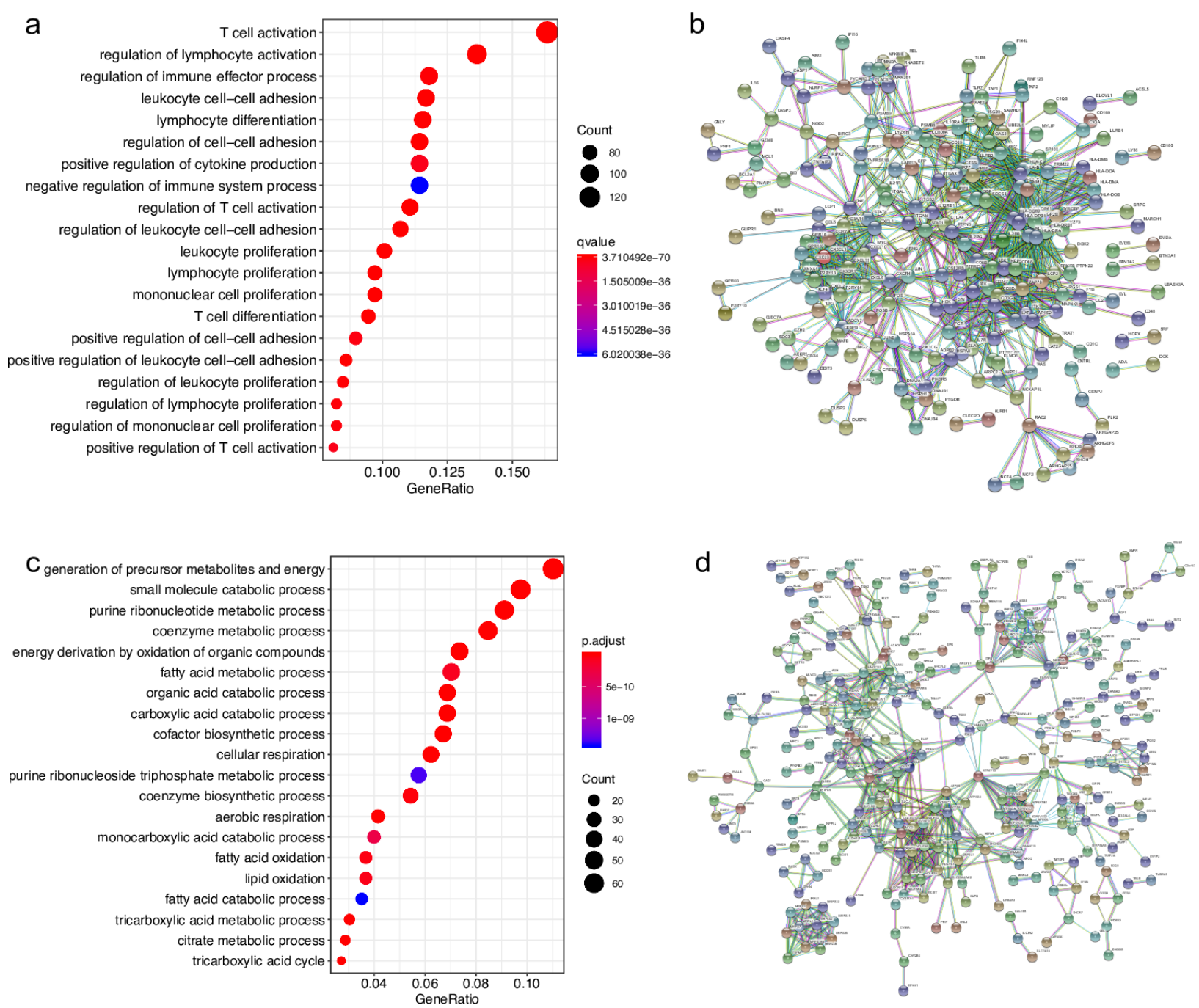

Fig S3. Pathway enrichment analysis of DE genes.

The top 20 GO terms enriched among (a) up- and (c) down-regulated genes in AR vs Normal analysis. Functional networking with STRING for the differentially expressed (b) up- and (d) down-regulated genes. We used the minimum required interaction score equal to 0.9 . The edges mean a type of interaction evidence. The colors mean as follows. The known interactions are cyan for curated databases and magenta for experimentally determined interactions. The predicted interactions are represented in green for gene neighborhood, red for gene fusions, and blue for gene co-occurrence. The other types of interactions are in lime based on text mining, black on co-expression, and indigo on protein homology. 


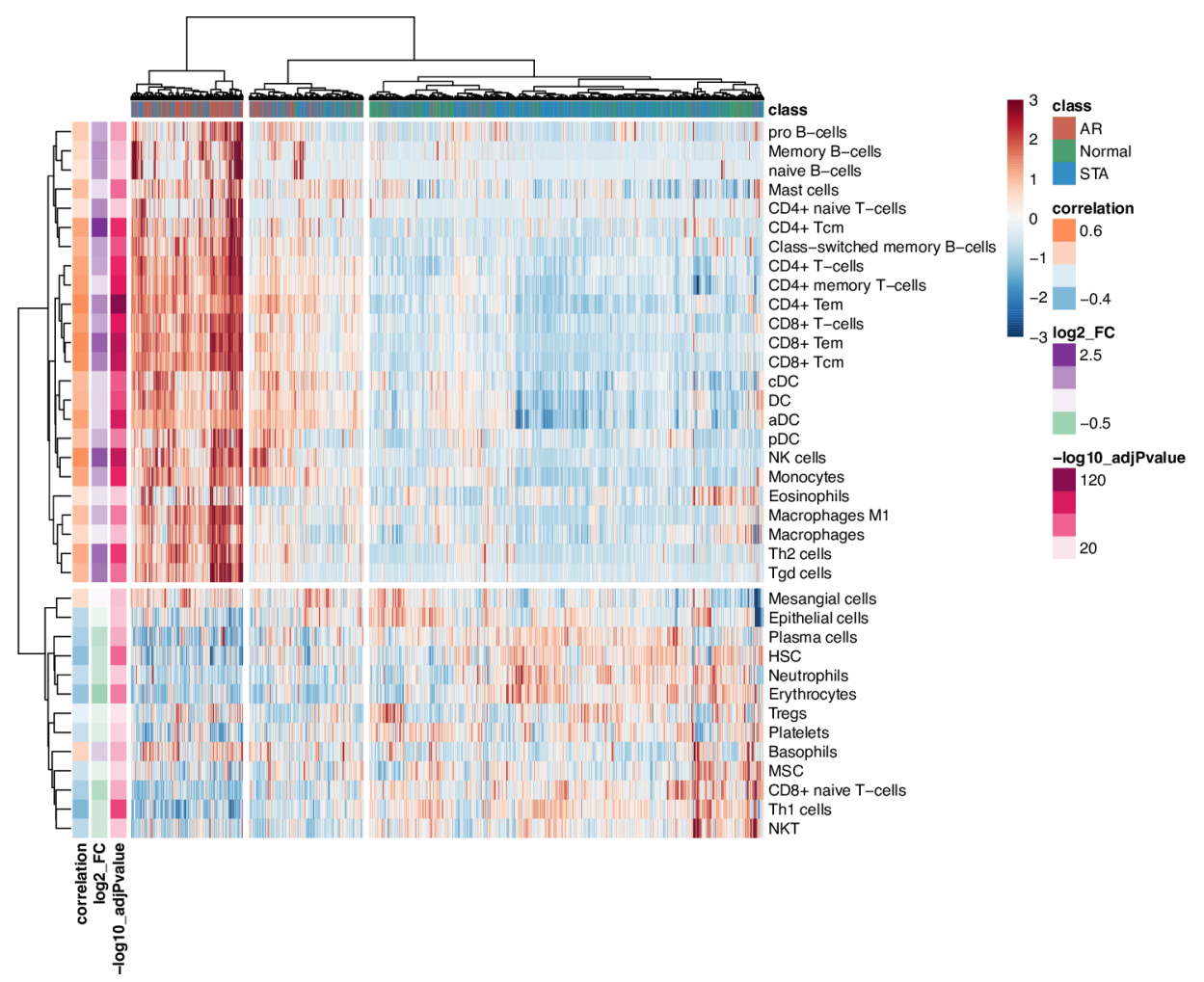

Fig S4. The heatmap of enrichment scores of significant cell types from the AR vs Normal comparison.

The colored vertical bars represent results from AR vs Normal analysis. The plot combines all AR, hSTA, and Normal samples and shows clustering of some hSTA samples together with AR hinting to possible hidden inflammation processes going in those grafts.
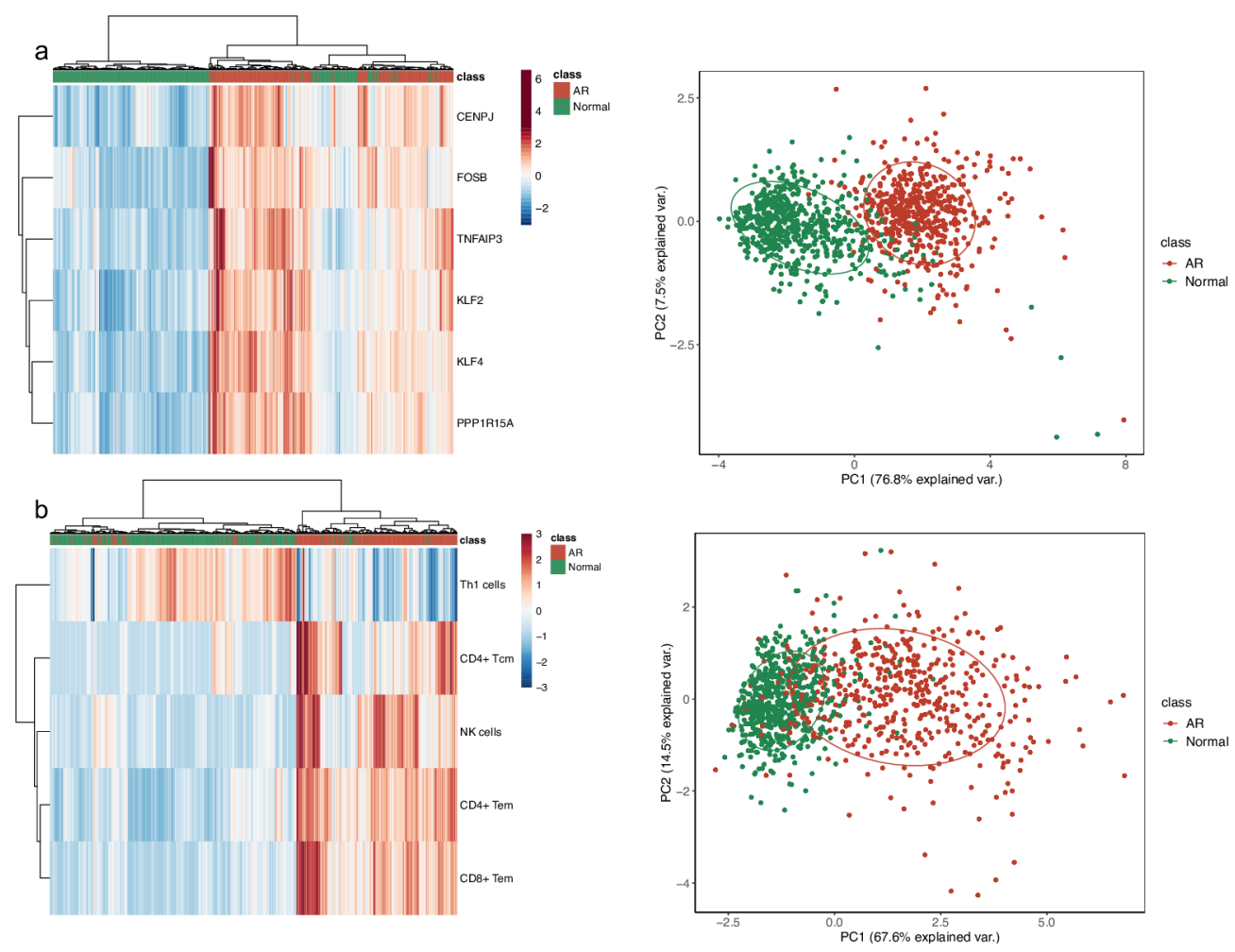
Fig S5. Plots of feature selected genes and cell types for all AR and Normal samples.

(a) Heatmap and PCA plot of feature selected gene expression. (b) Heatmap and PCA plot of feature selected cell types.

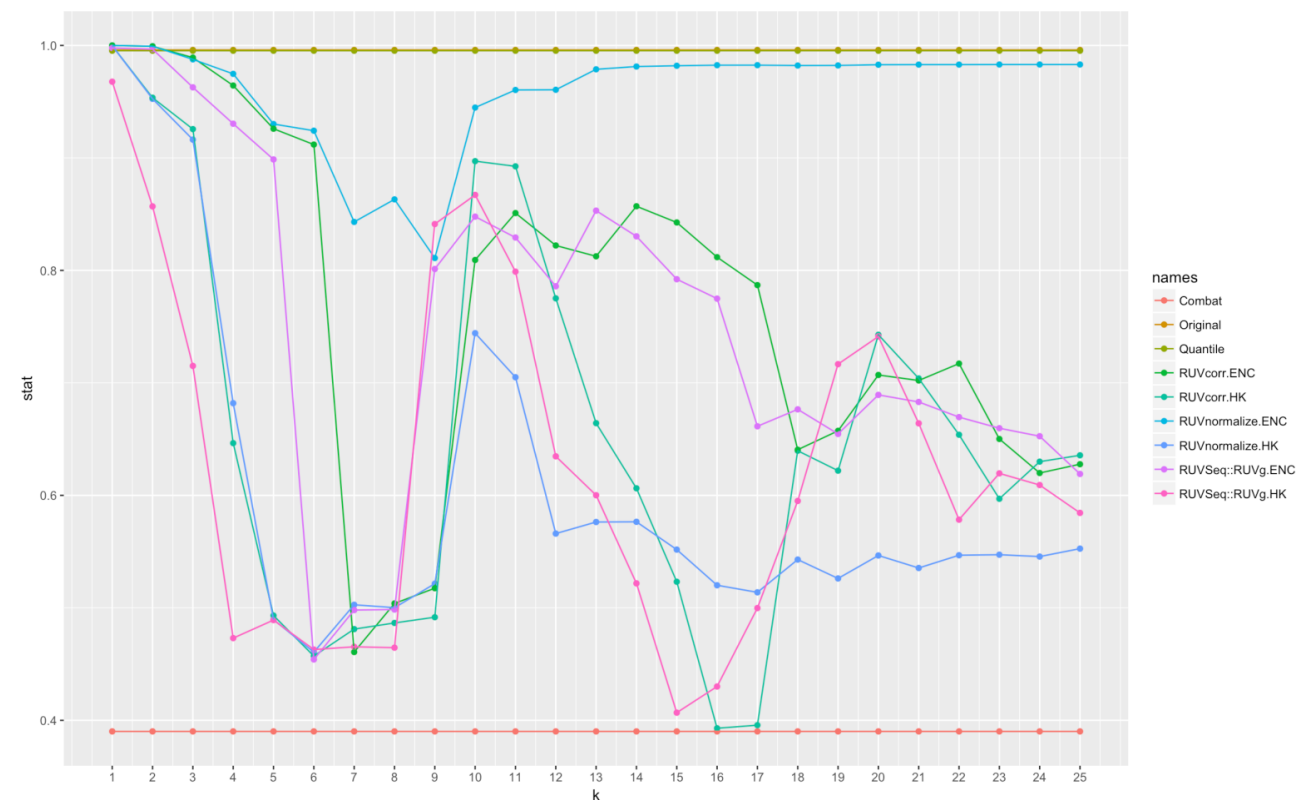

Fig S6. The combined banchmark based on p-value, delta statistic and the percentage of variability for batch correction methods tested.

$\mathrm{HK}$ - Housekeeping genes, ENC - Empirical Negative Control genes

\section{Supplementary Table}

$\begin{array}{rll}\text { Cells } & \text { Family } & \text { Type } \\ \text { Erythrocytes } & \text { HSC } & \text { HSC } \\ \text { HSC } & \text { HSC } & \text { HSC } \\ \text { Megakaryocytes } & \text { HSC } & \text { HSC } \\ \text { Platelets } & \text { HSC } & \text { HSC } \\ \text { B-cells } & \text { Immune } & \text { Lymphoid } \\ \text { CD4+ memory T-cells } & \text { Immune } & \text { Lymphoid } \\ \text { CD4+ naive T-cells } & \text { Immune } & \text { Lymphoid } \\ \text { CD4+ T-cells } & \text { Immune } & \text { Lymphoid } \\ \text { CD4+ Tcm } & \text { Immune } & \text { Lymphoid } \\ \text { CD4+ Tem } & \text { Immune } & \text { Lymphoid } \\ \text { CD8+ naive T-cells } & \text { Immune } & \text { Lymphoid } \\ \text { CD8+ T-cells } & \text { Immune } & \text { Lymphoid } \\ \text { CD8+ Tcm } & \text { Immune } & \text { Lymphoid } \\ \text { CD8+ Tem } & \text { Immune } & \text { Lymphoid } \\ \text { Class-switched memory B-cells } & \text { Immune } & \text { Lymphoid } \\ \text { Memory B-cells } & \text { Immune } & \text { Lymphoid } \\ \text { naive B-cells } & \text { Immune } & \text { Lymphoid }\end{array}$




\begin{tabular}{|c|c|c|}
\hline NK cells & Immune & Lymphoid \\
\hline NKT & Immune & Lymphoid \\
\hline Plasma cells & Immune & Lymphoid \\
\hline pro B-cells & Immune & Lymphoid \\
\hline Tgd cells & Immune & Lymphoid \\
\hline Th1 cells & Immune & Lymphoid \\
\hline Th2 cells & Immune & Lymphoid \\
\hline Tregs & Immune & Lymphoid \\
\hline $\mathrm{aDC}$ & Immune & Myeloid \\
\hline Basophils & Immune & Myeloid \\
\hline $\mathrm{cDC}$ & Immune & Myeloid \\
\hline DC & Immune & Myeloid \\
\hline Eosinophils & Immune & Myeloid \\
\hline iDC & Immune & Myeloid \\
\hline Macrophages & Immune & Myeloid \\
\hline Macrophages M1 & Immune & Myeloid \\
\hline Macrophages M2 & Immune & Myeloid \\
\hline Mast cells & Immune & Myeloid \\
\hline Monocytes & Immune & Myeloid \\
\hline Neutrophils & Immune & Myeloid \\
\hline $\mathrm{pDC}$ & Immune & Myeloid \\
\hline Epithelial cells & Non-Hematopoietic & Epithelial \\
\hline Endothelial cells & Non-Hematopoietic & Stroma \\
\hline Fibroblasts & Non-Hematopoietic & Stroma \\
\hline ly Endothelial cells & Non-Hematopoietic & Stroma \\
\hline Mesangial cells & Non-Hematopoietic & Stroma \\
\hline MSC & Non-Hematopoietic & Stroma \\
\hline nv Endothelial cells & Non-Hematopoietic & Stroma \\
\hline
\end{tabular}

Table S1. Cell types used in cell type enrichment analysis with xCell 


\section{Supplementary methods}

\section{Cross-study normalization in data merging}

We have examined several batch correction methods: ComBat [40] as a part of sva package [41], Quantile Normalization (QN) [42], Remove Unwanted Variation (RUV) [43] and Harman [44]. Currently, the most common technique to remove the systematic batch effects from biological data is ComBat [40] which based on empirical Bayes method to estimate batch effects and to adjust data across genes. However, often a major limitation of its use is missed: the studies merged have to be more or less balanced with respect to the case-control breakdown of samples. If it is not the case, some biological heterogeneity can vanish [36]. The breakdown by phenotype for each study we use in our work is represented in the Table 1 and shows presence of some imbalance in outcomes. Therefore, we wanted to additionally examine some other normalization methods comprehensively.

The Quantile Normalization technique is the same method used for normalization at the probe level, but as it was shown before [42] it can be applied for batch effect elimination as well.

The RUV method [43] is based on adjustment on so-called negative control genes, that are expected not to be differentially expressed across phenotypes. There is still an open question which set of genes would be most suitable for this role. Basically, so far there are two possible ways to obtain a list of control genes: housekeeping genes and empirically found ones. The housekeeping genes are defined to be expressed on the similar level across all tissues and involved in basic cell processes [104]. However, besides the fact that there is no fully established list of housekeeping genes [105], it was shown that they might not be the best to represent negative controls in adjustments [43] since they can be differentially expressed in some tissues [33] or related to diseases [106]. Another method is to empirically find genes that are expressed steadily across merging studies. However, there is some freedom in setting a number of such genes. Too small number can be not enough for proper adjustment, too large can include some genes important for a current study. Some dependence on studies involved is presented in the search for negative control genes. For some discussion of advantages and disadvantages of both methods see reference [107]. Another challenging factor in applying the RUV method is the parameters adjustment. Depending on implementation of the algorithm, there are two main parameters to adjust: the dimension of the unwanted systematic noise and the ridge smoothing parameter. To find an optimal set of parameters is actually a tricky problem. In our study, we examined three different implementations of RUV method in R packages RUVcorr [107], RUVnormalize [108], and RUVSeq [109] (RUVg function). The first two packages implement naïve RUV-random method which is a variation of the RUV-2 method originally described in [43]. The third method RUVg within RUVseq package is originally designed as a discrete version of RUV-2 methods for RNA-seq counts data. We used this method for performance comparison with other approaches. We compared the performance of these RUV implementations with the housekeeping and empirical negative control genes. We also varied the parameter of the noise dimension $k$ but set the smoothing ridge parameter to 0.001 for RUVnormalize and 0 for RUVcorr. 
Another promising normalization method we examined is Harman [44]. This method is a Principal Component Analysis based optimization technique that maximizes batch removal but keeps some probability of the overcorrection as a parameter. We compared the method performance with the overcorrection parameter set to 0.95 .

We considered three types of benchmarks to perform the comparison for normalization methods. We computed the percentage of variability in first ten principal components that can be explained by batch (i.e. by dataset study) and kept the maximum value among those ten as one of three benchmarks. For the other two we used the R package gPCA [110] to compute guided principal components (i.e. the principal components of batch modified data) and obtained a p-value (i.e. the probability of having batch effect in data) and delta statistic (i.e. the ratio of guided and unguided first principal component; the lower the better). Since all three metrics are in the range from 0 to 1 , we summed them up and normalized to one and used as a final metric to justify the normalization method performance. The results are represented in the Supplementary Fig S6.

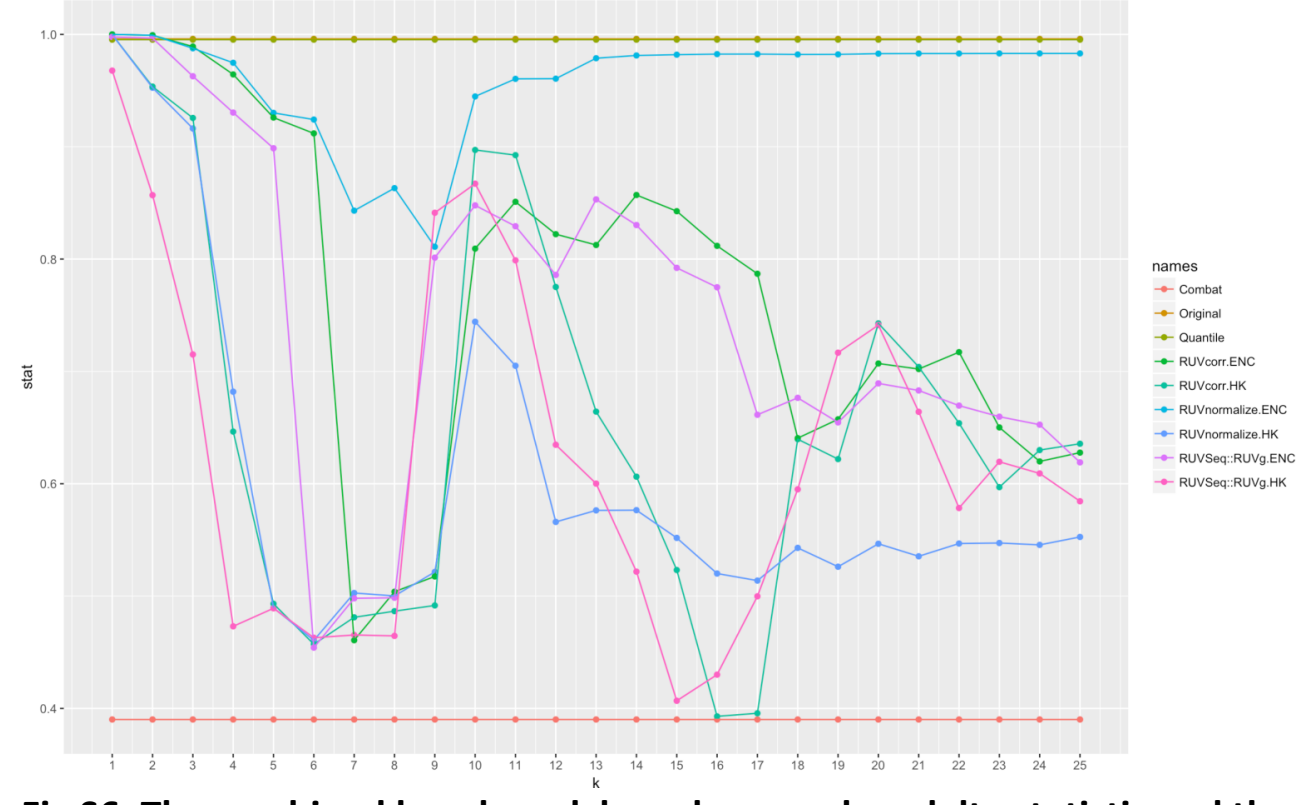

Fig S6. The combined banchmark based on p-value, delta statistic and the percentage of variability for batch correction methods tested.

$\mathrm{HK}$ - Housekeeping genes, ENC - Empirical Negative Control genes

We found the Harman normalization to outperform all methods followed by ComBat and RUV implementations in RUVcorr and RUVseq with housekeeping genes at the noise dimension parameter $k=15-17$. Further comparison analysis showed that while the data seems batch free, RUV and Harman adjusted a bit too much leaving minimum of biological variability, resulting in much fewer differentially expressed genes in comparison to ComBat. Therefore, we decided to use ComBat for cross-study normalization to keep as much heterogeneity as possible while successfully adjusting for batch effects. We observed that the results of the normalization performance vary depending on datasets: their number, platform, processing methods. There is no one-fit-all technique that should be used blindly to correct for batch effects when merging 
bioRxiv preprint doi: https://doi.org/10.1101/2019.12.18.881706; this version posted December 19, 2019. The copyright holder for this preprint (which was not certified by peer review) is the author/funder, who has granted bioRxiv a license to display the preprint in perpetuity. It is made available under aCC-BY-ND 4.0 International license.

data. Therefore, it should be advised to perform such comparisons of normalization methods each time performing meta-analysis to choose best among available methods [35]. 